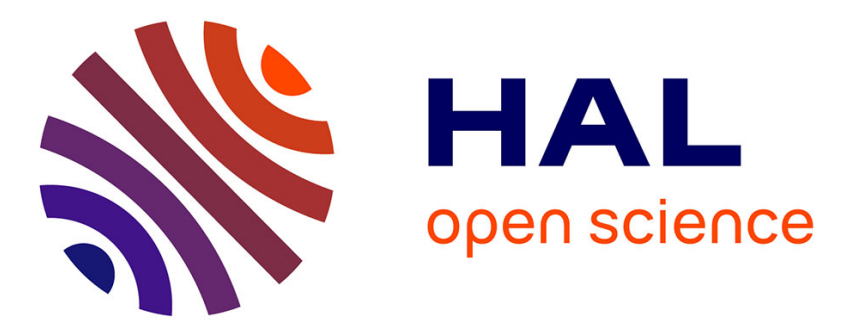

\title{
The NC-CC Isotope Dichotomy: Implications for the Chemical and Isotopic Evolution of the Early Solar System
}

Katherine R Bermingham, Evelyn Füri, Katharina Lodders, Bernard Marty

\section{- To cite this version:}

Katherine R Bermingham, Evelyn Füri, Katharina Lodders, Bernard Marty. The NC-CC Isotope Dichotomy: Implications for the Chemical and Isotopic Evolution of the Early Solar System. Space Science Reviews, 2020, 216 (8), pp.133. 10.1007/s11214-020-00748-w . hal-03321435

\section{HAL Id: hal-03321435 \\ https://hal.science/hal-03321435}

Submitted on 17 Aug 2021

HAL is a multi-disciplinary open access archive for the deposit and dissemination of scientific research documents, whether they are published or not. The documents may come from teaching and research institutions in France or abroad, or from public or private research centers.
L'archive ouverte pluridisciplinaire HAL, est destinée au dépôt et à la diffusion de documents scientifiques de niveau recherche, publiés ou non, émanant des établissements d'enseignement et de recherche français ou étrangers, des laboratoires publics ou privés. 


\title{
The NC-CC Isotope Dichotomy: Implications for the Chemical and Isotopic Evolution of the Early Solar System
}

\author{
Katherine R. Bermingham ${ }^{1,2} \cdot$ Evelyn Füri ${ }^{3}$ Katharina Lodders ${ }^{4} \cdot$ Bernard Marty $^{3}$ \\ 1 Department of Earth and Planetary Sciences, Rutgers University, Piscataway, NJ 08854, USA \\ 2 Department of Geology, University of Maryland, College Park, MD 20742, USA \\ 3 Université de Lorraine, CNRS, CRPG, 54000 Nancy, France \\ 4 Department of Earth and Planetary Sciences and McDonnell Center for the Space Sciences, Washington \\ University in St. Louis, St. Louis, MO 63130, USA
}

This is a pre-print of an article published in Space Science Reviews. The final authenticated version is available online at: https://link.springer.com/article/10.1007/s11214-020-00748-w

\begin{abstract}
Understanding the formation of our planetary system requires identification of the materials from which it originated and the accretion processes that produced the planets. The compositional evolution of the solar system can be constrained by synthesizing astronomical datasets and numerical models with elemental and isotopic compositions from objects that directly sampled the disk: meteorites and their constituents (chondrules, refractory inclusions, and matrix). This contribution reviews constraints on early solar system evolution provided by the so-called non-carbonaceous (NC) and carbonaceous chondrite (CC) groups and their relationship to the volatile element characteristics of chondritic meteorites. In previous work, the $\mathrm{NC}$ or $\mathrm{CC}$ character of a parent body was used to infer its accretion location in the protoplanetary disk. The NC groups purportedly originated in the inner disk, and the $\mathrm{CC}$ groups were derived from the outer disk, where the $\mathrm{NC}$ and $\mathrm{CC}$ regions of the disk may have been separated early on by proto-Jupiter, a pressure maximum, or a dust trap in the disk. The tenet that all $\mathrm{CC}$ parent bodies accreted in the outer disk is, in part, based on evidence that a handful of CC meteorites are enriched in volatile species compared to NC meteorites. Here, it is reviewed if and how the volatile element and nucleosynthetic isotope compositions of meteorites can be linked to accretion locations within the disk. The nucleosynthetic isotope compositions of whole rock meteorite samples contrast the trends found for their major volatile element compositions (i.e., C, N, and O). Although there may be an increase in volatile abundances when comparing some stony $\mathrm{NC}$ and $\mathrm{CC}$ meteorites and their inferred accretion locations within the disk, this is not necessarily a general rule. The difficulties with inferring parent body accretion locations are discussed. It is found that it cannot always be assumed that parent bodies which formed in the $\mathrm{CC}$ reservoir are "volatile-rich" relative to those that formed in the NC reservoir which are "volatilepoor". Consequently, tracing the origin of terrestrial volatiles using the $\mathrm{NC}-\mathrm{CC}$ isotope dichotomy remains challenging.
\end{abstract}

Keywords NC-CC; Volatiles; Nucleosynthetic isotope anomalies; Meteorites; Protoplanetary disk evolution 


\section{Introduction to Solar System Formation}

The solar system formed from the solar nebula, which was a segment of a molecular cloud made of interstellar gas and dust particles that had spun into a disk around the forming Sun. This includes dust from stellar sources that was added to the interstellar medium at least $\sim 3 \pm 2 \mathrm{Ga}^{1}$ before the birth of the solar system (e.g., Heck et al. 2020). The solar nebula separated from the main molecular cloud mass due to gravitational collapse of the cloud which was caused by a sufficient weakening of magnetic field support following ambipolar diffusion (Shu et al. 1987) or by shockwaves from nearby supernova (Cameron and Truran 1977; Boss 1995). Due to conservation of angular momentum, the solar nebula formed a rotating disk (generally referred to as the protoplanetary disk) around the central protostar. During the earliest stage of the protoplanetary disk, angular momentum was transported outward, while significant mass was transported inward and accreted onto the protostar or was lost by FUV photoevaporation (e.g., Williams and Cieza 2011).

The oldest preserved solids thought to have condensed first in the protoplanetary disk are calciumaluminum-rich inclusions (CAIs), which have an average absolute $\mathrm{U}$-corrected $\mathrm{Pb}-\mathrm{Pb}$ age of 4,567.30 \pm 0.16 Ma (Connelly et al. 2012). This age is the cosmochemical start $\left(\mathrm{t}_{0}\right)$ of the solar system. Within $<1$ Ma of CAI formation, planetesimals began to accrete throughout the protoplanetary disk (e.g., Kruijer et al. 2014; Sugiura and Fujiya 2014). Some planetesimals had sufficient concentrations of heat-producing radioactive isotopes (primarily ${ }^{26} \mathrm{Al}$ ) to cause melting, differentiation, and formation of a metallic core and a silicate mantle and crust (Lee et al. 1977; Shukolyukov and Lugmair 1993a, 1993b; Hevey and Sanders 2006; Sahijpal et al. 2007; Moskovitz and Gaidos 2011). Collisional accretion and subsequent oligarchic growth of planetesimals gave rise to the evolution of planetesimals into a planetary system (for a recent review, see Walsh and Levison 2019).

Presently, a gross zonation in chemistry and mass is exhibited by the planets, where the outer giant planets (Jupiter, Saturn, Uranus, Neptune) are gas- and ice-rich, ${ }^{2}$ whereas the inner rocky planets (Mercury, Venus, Earth, Mars) are relatively depleted in highly-volatile and volatile elements. In part, the chemical gradient is considered to be a consequence of clearing gas from the inner solar system by early solar activity, rapid growth of the giant planets, and incomplete condensation from the protoplanetary disk (for a review, see Taylor 2001; Lodders and Fegley 2011; Palme et al. 2014; Cieza et al. 2016, and references therein). The ice to rock ratio favors a larger mass of ice in cooler regions in planetary accretion disks that enables rapid growth of gasgiant planets and provides the critical mass necessary to allow efficient gravitational capture of nebular gas (e.g., Wasson 1988). This provides an explanation for the H- and He-rich atmospheres of the outer planets and their much larger masses than the terrestrial planets. Observations of nearby planetary systems indicate that these planetary forming processes are not unique to our solar system (e.g., Cieza et al. 2016; Kudo et al. 2018).

Despite understanding the generalities of planet formation, important questions remain. For example, what was the composition of the disk and how did it evolve? To what extent are the different stages of disk evolution and planetary accretion recorded by the composition of the planets, asteroids, and meteorites? Can these cosmochemical materials be used to accurately reconstruct the formation of the solar system? What was the volatile element distribution throughout the disk and how did it change with planet growth? What was the source of water and other volatiles to Earth?

Insights to these questions come from analyzing material that directly sampled the proto- planetary disk: chondritic, achondritic, and iron meteorites. Recent developments in analytical instrumentation have made it possible to determine the subtle (on the parts per million scale) isotopic variations between bulk meteorites and their individual components (Figs. 1 and 2).

\footnotetext{
1 The IUPAC-IUGS convention for expressing dates following recommendation by Holden et al. (2011) is that annus (a) is used for one year as a unit of time, both for absolute time and time differences. Hence, considering the declensions, it is $\mathrm{Ga}=$ Giga annis (ablative for age; used as appellative) or $\mathrm{Ga}=$ Giga annos(pl. accusative, for how long ago).

2 The enrichments of $\mathrm{C}, \mathrm{N}$, and $\mathrm{O}$ (relative to solar) observed in outer planet atmospheres were caused by the accretion of ices bearing these highly volatile elements. Highly volatile elements have $50 \%$ condensation temperatures below $371 \mathrm{~K}$, volatile elements below $665 \mathrm{~K}$, moderately volatiles between 1335 and $665 \mathrm{~K}$, and refractories above $1335 \mathrm{~K}$ (for a gas of solar composition at a total pressure of $10^{-4}$ bar; Lodders 2003).
} 


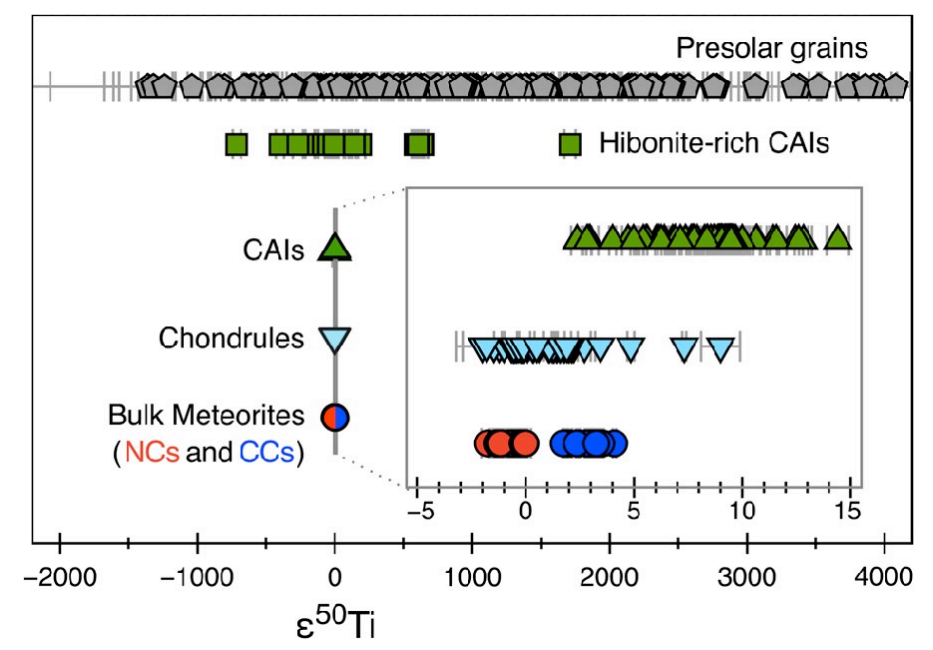

Fig. 1 Variation in ${ }^{50} \mathrm{Ti} /{ }^{47} \mathrm{Ti}$, expressed in $\varepsilon$ units (parts per 10,000; see Sect. 3 for details), in presolar grains, CAIs, chondrules, and "bulk" CC- and NC-type meteorites (chondrites and achondrites). The inset illustrates that CAIs, chondrules, and bulk meteorites record small $\varepsilon^{50}$ Ti variations ( $<20 \varepsilon$ units) compared to presolar grains ( $\geq 5000 \varepsilon$ units, corresponding to $500 \%$ in $\delta$ units). Error bars are smaller than symbol sizes in most cases, except for presolar grains. Modified from Torrano et al. (2019) with data from the literature (Alexander and Nittler 1999; Amari et al. 2001; Davis et al. 2018; Gerber et al. 2017; Gyngard et al. 2018; Hoppe et al. 1994; Huss and Smith 2007; Ireland et al. 1991; Kööp et al. 2016; Niemeyer 1988; Render et al. 2019; Torrano et al. 2019; Trinquier et al. 2009; Zhang et al. 2012; Zinner et al. 2007).

Here, isotopic variations are defined as isotopic deviations from a terrestrial composition. ${ }^{3}$ In parallel, attempts have been made to relate meteorite isotopic and volatile compositions to their parent body's accretion location in the protoplanetary disk, and to use models to reconstruct the architecture of the evolving protoplanetary disk (e.g., Warren 2011; Kruijeret al. 2017). This approach relies on the assumptions that: (1) the terrestrial meteorite collection is representative of the entire or a substantial population of planetesimals present during accretion; (2) the elemental and isotope composition of a meteorite group faithfully represents the composition of the disk region from which its parent body(ies) accreted; (3) the volatile content of meteorites accurately records the volatile content of the parent body and the disk region it sampled; (4) the combination of a meteorite's isotope composition and the volatile element content can trace the communication between different regions of the disk. This contribution reviews work that uses the composition of meteorites to deduce the evolution of the protoplanetary disk from dust to the planetary system observed today. It begins with a brief review of meteorites and their components. This is followed by an outline of isotopic variations in meteorites, meteorite volatile content, parent body accretion locations, and the applicability of these data to reconstructing the evolution of the disk and, finally, the accretion history of Earth. We present several old and new ideas without claiming to be comprehensive. Our aim is to make the reader aware that, despite the recognition of the presence of a dichotomy in isotopic composition, a synthesis of perspectives and data to produce a unique explanation of the origin of all elemental and isotopic peculiarities is still under development. This state is reflected in the primary literature, which often focuses on subsets of specific isotopic systems and/or particular theoretical approaches but does not always include a rigorous assessment of existing and/or other new ideas. In time, a clearer picture will likely emerge when sample-based data are more completely understood and integrated with astronomical datasets and numerical models.

\footnotetext{
3 Typically, isotope ratios are measured, and meteorite samples with an excess or depletion of a given isotope will have a higher or lower (respectively) isotope ratio than the terrestrial reference value. Positive or negative isotope anomalies arise from the definition of the isotope notation, which is the difference between the measured sample ratio from that of the terrestrial standard value in percent (\%), permil (\%), on a scale of parts per 10,000 $(\varepsilon$ units), or in parts per 1,000,000 ( $\mu$ units). The advantage of using the differences in the ratios is that these scales are easier to work with, especially for samples with small isotope anomalies (bulk meteorites, chondrules, CAIs). Terrestrial materials are generally defined as 'normal' (or equal to zero) in isotopic composition.
} 


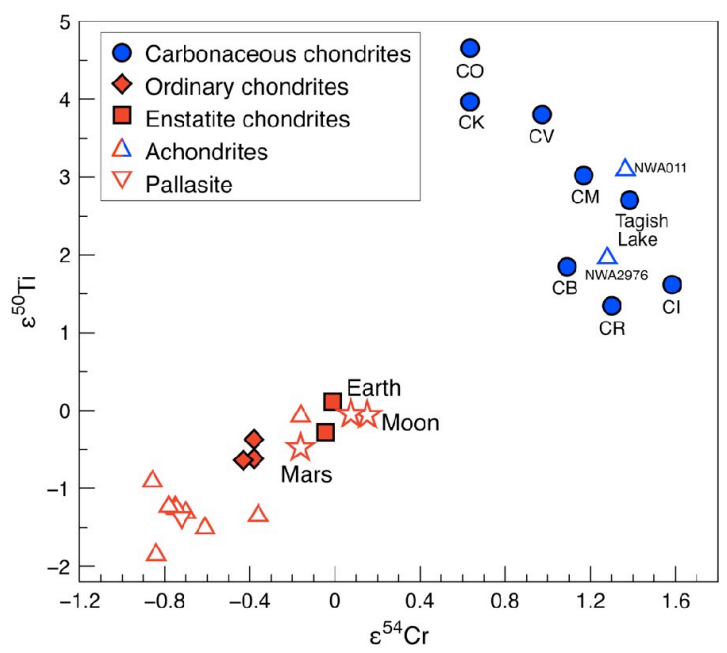

Fig. 2 Variation in ${ }^{50} \mathrm{Ti} /{ }^{47} \mathrm{Ti}$ and ${ }^{54} \mathrm{Cr} / 52 \mathrm{Cr}$, expressed in $\varepsilon$ units (see Sect. 3 for details), between undifferentiated (filled symbols) and differentiated meteorites (open symbols) in comparison to Earth, Moon, and Mars (star symbols). CCand NC-type bodies are represented in blue and red, respectively. Modified from Warren (2011) and Scott et al. (2018), and datasources therein.

\section{Meteorites and Their Components}

Most meteorites originate from the asteroid belt, ${ }^{4}$ which is a low mass region (representing $\sim 4 \%$ of the mass of the Moon) of the solar system between the orbits of Mars and Jupiter. The asteroid belt provides the most direct sampling of the protoplanetary disk via meteorites delivered to Earth. The belt has a gross chemical stratification, with an inner region that is dominated by silicaceous (S-type) asteroids and an outer region dominated by dark carbonaceous (C-type) asteroids. While some material in the asteroid belt presumably accreted in situ, the diversity of meteorite compositions (ranging from dry, igneous rocks to 'comet- like' icerock mixtures) indicates that the asteroid belt received material originating from the "inner" and "outer" solar system. Here, the material inboard of Jupiter is defined to come from the inner solar system and material outboard of Jupiter originated from the outer solar system (following Warren 2011). It has been proposed that some asteroids were scattered in- wards and outwards during the growth and/or migration of the gas giants (Walsh et al. 2011; Warren 2011; Morbidelli et al. 2015; for a recent review, see Raymond et al. 2020). Hence, the present distribution of asteroids is unlikely reflective of their original accretion location. Meteorites are from either melted (achondrites, stony irons, and irons) or unmelted, 'primitive' (chondrites) parent bodies. 'Parent body' refers to either a planet, moon, or asteroid. Achondrites and iron meteorites sample the silicatedominated crust/mantle and metallic core, respectively, of bodies that experienced large-scale melting and differentiation. It should be noted that iron meteorites are not chemically or isotopically linked to known achondrites, which suggests that the silicate mantles of iron meteorite parent bodies are "missing" and might have been destroyed by impacts in the early solar system. Also, there is a lack of achondrites with the expected mineralogy and chemical composition of mantles of differentiated planetesimals (except for martian and lunar meteorites).

Main group pallasites (so-called stony irons, composed of olivine and Fe-Ni-rich metal) had long been thought to represent the transition zone between the core and mantle of differentiated asteroids (Scott 1977). The highly siderophile element abundances of pallasites are consistent with late crystallization of a metallic core, as expected near the core- mantle boundary (Scott 1977; Davis 1977). Updated metallographic cooling rates and chemical compositions, however, indicate an impact origin for these meteorites (Yang et al. 2007, 2010). Recent high strain-rate deformation experiments were used to identify the process of pallasite formation, and results indicated that pallasites could form via two-stages:

\footnotetext{
${ }^{4}$ Additional sources of achondrites to Earth include Mars and the Moon. The meteorites referred to here do not include micrometeorites.
} 
(1) inefficient core-mantle differentiation, and (2) an impact event (Walte et al. 2020). Chondrites sample planetesimals that have not experienced wholesale melting and differentiation. As such, they are often referred to as "primitive" meteorites, and their compositions may reflect the composition of the disk material from which they accreted. This does not mean they record the exact state of the primitive condensable materials that were present in the solar nebula because all chondrites underwent metamorphic (aqueous and/or thermal) processing on their assembled parent bodies. Nonetheless, compared to planets, satellites, and achondrites, chondrites represent the least altered materials from the solar nebula which can be studied.

Based on mineralogical, chemical, and isotopic differences, chondrites are divided into three major classes: carbonaceous, ordinary, and enstatite chondrites (Fig. 2), and these classes are further divided into chondritic groups (Fig. 3). There are substantial mineralogical and petrographic characteristics as well as whole-rock chemical and O-isotopic compositions which support the interpretation that chondrite groups sample separate parent bodies (for review, see Weisberg et al. 2006). Spectroscopic observations link enstatite/ordinary and carbonaceous chondrites to asteroids in the inner and outer belt, respectively, (e.g., Morbidelli et al. 2012, and references therein). Vernazza et al. (2014, 2016) and Patzer and Schultz (2002), however, questioned if each chondrite group represents a single distinct parent body.

Chondritic meteorites consist of three main components: refractory inclusions (CAIs, hibonite-rich CAIs, and amoeboid olivine aggregates), chondrules, and matrix (e.g., Hezel et al. 2019; Krot 2019). Recent advances have established a chronology of formation of CAIs and chondrules in the context of plausible formation models that account for their chemical and isotopic compositions. Evidence from short- and longlived isotopes, oxy- gen isotopes, nucleosynthetic isotopic anomalies, and petrologic studies suggest that CAIs were the first solids to have formed in the protosolar disk (for a review, see MacPherson 2014). The oxygen isotopic compositions of CAIs suggest that they formed in regions that were predominantly solarlike and ${ }^{16}$ O-rich compared to Earth (e.g., MacPherson 2014). Thermochemical modelling of the condensation of refractory inclusions indicates that the main minerals in CAIs condensed in a low pressure, high temperature $(>1300 \mathrm{~K})$ environment from a solar composition gas (which had an oxygen fugacity several orders of magnitude below the iron-wüstite buffer, IW) (Grossman and Larimer 1974; Lodders 2003). There are, however, indications from trace element abundance patterns that the oxygen fugacity during condensation was variable (e.g., Fegley and Palme 1985; Rubin et al. 1988).

Chondrules, the dominant component in most chondrites, are up to millimeter-sized silicate-rich spherules that had been partially or completely molten prior to incorporation into a planetesimal (Scott and Krot 2005). They preserve mineral compositions and textures that indicate crystallization within minutes to hours (Krot et al. 2018 and references therein). ${ }^{26} \mathrm{Al}-{ }^{26} \mathrm{Mg}$ systematics indicate that most chondrules formed 1-3 Ma after CAIs (e.g., Villeneuve et al. 2009; Pape et al. 2019). This conclusion is contrary to $\mathrm{Pb}-\mathrm{Pb}$ ages that indicate some chondrules formed contemporaneously with CAIs which would imply that chondrules record a heterogeneous distribution of the short-lived ${ }^{26} \mathrm{Al}$ nuclide in the protoplanetary disk (Connelly and Bizzarro 2017). The chemical and mineralogical characteristics of chondrules indicate that they formed under diverse physico-chemical conditions in the nebula that were generally more oxidizing, at higher total pressures and/or higher dust:gas ratios than the CAI-forming region.

Chondrite matrices are volatile-rich compared to refractory inclusions and chondrules. Matrices are characterized by fine-grained mixtures of materials with diverse origins in the protoplanetary disk. This finegrained mixture can be annealed during parent body thermal metamorphism. In objects that have not undergone thermal metamorphism, the matrix generally shows signs of aqueous alteration, e.g., from the presence of secondary mineral phases such as carbonates, sulfates, and phyllosilicates (for a review, see Brearley 2006). Matrices also house primary constituents that formed in the disk, including broken fragments of hightemperature components (e.g., CAIs and chondrules) and low-temperature phases (e.g., organic materials). 

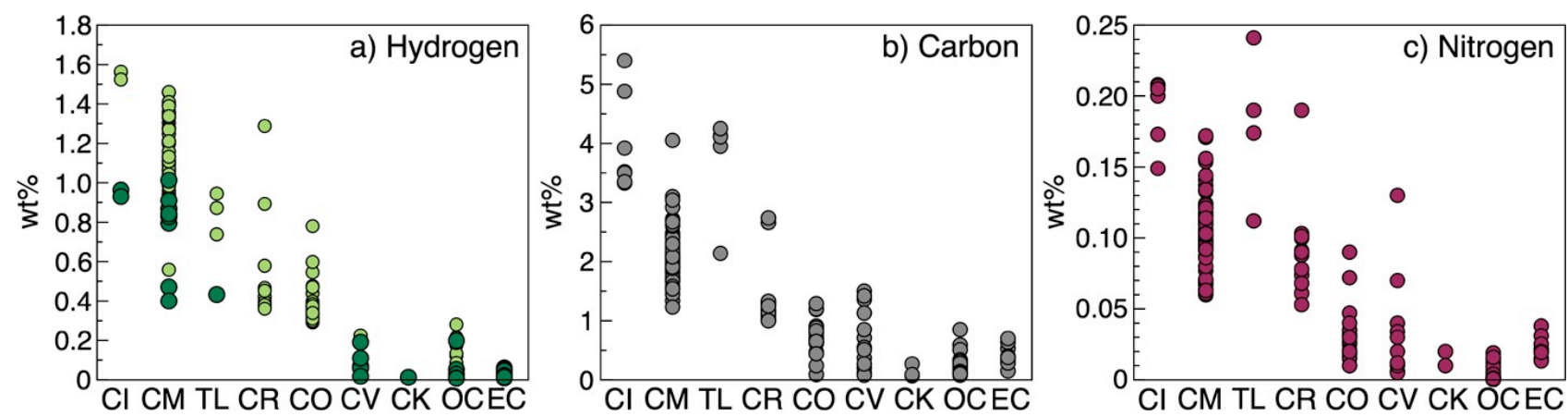

Fig. 3 (a) Hydrogen, (b) carbon, and (c) nitrogen content (in wt\%) of carbonaceous (CI, CM, Tagish Lake (TL), CR, CO, CV, CK), ordinary (OC), and enstatite chondrites (EC). Hydrogen abundances from Vacher et al. (2020) (dark green) are lower, in most cases, than those from Alexander et al. $(2012,2018)$ (light green) as a result of pre-degassing and removal of atmospheric contamination under vacuum at $120^{\circ} \mathrm{C}$ for $48 \mathrm{~h}$ before measurement. Hydrogen abundances of ECs are from Piani et al. (2020). Carbon and nitrogen concentrations are from Alexander et al. (2012, 2018), Grady et al. (1986), Hashizume and Sugiura (1995), and Pearson et al. (2006).

Chondrite matrices can also preserve presolar grains and insoluble/refractory organics (e.g., Lodders and Amari 2005; Zinner 2014; Floss and Haenecour 2016; Nittler and Ciesla 2016; Takigawa et al. 2018). Presolar grains formed in stellar outflows or ejecta and remained intact throughout their journey into the solar system where they were preserved in the disk (Lodders and Amari 2005). Since presolar grains incorporate the dominant isotope products of nucleosynthetic reactions operating in their parent star, their isotope compositions are highly variable and well outside the range found in solar system materials (Fig. 1). Presolar grains exhibit diverse mineralogy and include $\mathrm{SiC}$, graphite, carbides or metal grains in graphite, $\mathrm{Si}_{3} \mathrm{~N}_{4}$, corundum, spinel, hibonite, $\mathrm{TiO}_{2}$, and amorphous and crystalline silicates (for review, see Zinner 2014; Floss and Haenecour 2016; Nittler and Ciesla 2016). Preservation of these grains in some meteorites is a testament to their chemical robustness and ability to withstand the process of disk formation and evolution, and parent body accretion and alteration. Differentiated planetesimals presumably formed from material which included presolar grains; however, these grains are not pre- served in achondrites and irons because the extensive processes of heating and melting (and potentially the low oxygen fugacity) destroyed them.

\section{Nucleosynthetic Isotope Variations in the Protoplanetary Disk}

Historically, the protoplanetary disk was considered to be a hot, compositionally homogeneous disk of dust and gas (e.g., Urey 1952). The understanding of the composition of the disk changed considerably with the study of meteorites. The discovery of isotopic variations in xenon (Reynolds and Turner 1964), neon (Black and Pepin 1969), N, Si, C (Lewis et al. 1987; Zinner et al. 1987), and SiC grains (Bernatowicz et al. 1987) in meteorites led to the realization that products of precursor stars were preserved in the solar system. These observations required that the protoplanetary disk did not reach temperatures high enough to vaporize (and homogenize) all presolar grains. Following this and the discovery of oxygen isotopic variations in meteorites ${ }^{5}$ (Clayton et al. 1973), the concept of a cooler, isotopically heterogeneous disk that retained a cosmic chemical memory of the solar system's parental molecular cloud was advanced (e.g., Clayton 1982).

Subsequent isotope measurements of meteorites revealed variations in individual components and bulk samples that could not be explained by mass-dependent (physical or chemical) processes, radioactive decay, cosmic ray exposure effects, or nuclear field shift effects. Generally, bulk sample isotopic compositions of meteorites are less anomalous than those found in individual presolar grains, which is likely due to dilution of the presolar grain compositions by disk dust (Fig. 1). By contrasting isotope compositions of bulk meteorite samples with the much larger isotopic anomalies recorded by presolar grains, it was proposed that bulk sample isotopic variations were caused by the heterogeneous distribution of presolar grains in the protoplanetary disk 
(for review, see Qin and Carlson 2016). These isotopic variations were named nucleosynthetic isotope anomalies.

To date, meteoritic nucleosynthetic anomalies have been recorded in isotopes of $\mathrm{Ne}, \mathrm{Kr}, \mathrm{Xe}, \mathrm{Ca}, \mathrm{Ti}, \mathrm{Cr}$, Ni, Sr, Zr, Mo, Ru, Pd, Ba, W, Nd, Sm (e.g., Warren 2011; Ott 2014; Mayer et al. 2015; Bermingham et al. 2016; Dauphas and Schauble 2016; Qin and Carlson 2016; Kruijer et al. 2017; Bermingham et al. 2018a, 2018b). Bulk meteorite isotopic variations are generally considered to reflect the bulk (or average) composition of the parent body, and thus the nebular region from which it accreted (e.g., Walker et al. 2015). The small scale of nucleosynthetic isotope anomalies indicates that the protoplanetary disk became reasonably, but not perfectly, homogenized during its evolution. Consensus on the process(es) that led to the heterogeneous distribution of presolar grains in the disk is yet to be reached. Leading theories suggest it is the result of poor mixing of these grains into the protoplanetary disk (Clayton 1982 and references therein) and/or their selective destruction via thermal chemical processing in the protoplanetary disk (Trinquier et al. 2009).

\section{Can Meteorite Compositions Be Used to Constrain the Evolution of the Protoplanetary Disk?}

As most meteorites are samples of disk material that have been preserved for $\sim 4.56 \mathrm{Ga}$, their chemical and isotopic compositions can help reconstruct the physico-chemical evolution of the protoplanetary disk. If nucleosynthetic isotope signatures exhibited by bulk meteorites reflect the unique regional characteristics of the disk from which their parent bodies accreted, these isotopic variations could be used to trace heterogeneities in different regions of the disk (e.g., inner vs. outer solar system) and communication between these regions. Furthermore, if a robust link can be found between the nucleosynthetic isotope composition ofa parent body, its accretion location, and the amount of volatiles (e.g., H, C, N) it accreted, the source of water and other volatiles for Earth and the terrestrial planets could potentially be traced.

\subsection{Relating Meteorite Compositions to Accretion Locations in the Disk}

It has been suggested that chondrite properties (e.g., bulk sample oxidation state, oxygen isotopic composition, volatile and refractory lithophile element abundance, matrix/chondrule modal abundance ratio) varied with heliocentric distance, and each chondrite group represents a different parent asteroid formed in a distinct nebular region and/or at a particular time (e.g., Wasson 1985, 1988; Rubin and Wasson 1995; Gradie and Tedesco 1982; Wood 2005; Raymond et al. 2009). The early article by Wasson (1988) argued for spatially separated formation locations for the different chondrite groups. The author proposed a model for forming the carbonaceous chondrites at the outer edge of the asteroid belt and the enstatite chondrites closest to the Sun $(<1$ $\mathrm{AU})$, and the ordinary chondrites in-between. Based on chemical and physical properties, it is now generally assumed that the parent bodies of enstatite chondrites formed closest to the Sun, whereas ordinary and carbonaceous chondrites sample planetesimals that accreted at increasing heliocentric distances (e.g., Rubin and Wasson 1995; Wood 2005). First, the proportion of chondrules in enstatite and ordinary chondrites has been interpreted to indicate that their parent bodies formed in the inner disk, where higher shock velocities than in the outer disk resulted in higher concentrations of chondrules (e.g., Wood 2005). The differences in chondrule chemistry, however, still require spatially separated accretion locations with different oxidation conditions, where resorting and concentrating chondrules alone is not a sufficient explanation. Second, the abundance of water and other volatiles in unequilibrated chondrites has been used as an approximate indicator of the radial distance of parent body accretion, thereby suggesting that carbonaceous chondrites formed in the outer protoplanetary disk (near or beyond the snow line; Wasson 1988; Sect. 5.2), where they were able to accrete abundant organic- and ice-rich components (e.g., Morbidelli et al. 2012; Trigo-Rodríguez et al. 2019). The formation of carbonaceous chondrites in the outer part of the protoplanetary disk was reinforced by reflection spectroscopy of objects in the outer asteroid belt (see Wasson 1988) and beyond (objects between Jupiter and Uranus, the irregular satellites of the giant planets, comets, and Kuiper belt objects) (e.g., Cloutis et al. 2012a,

5 Proposals for the origin of meteoritic oxygen isotopic variations now include photochemical effects (Clayton 2002) and non-mass dependent isotopic effects (Thiemens and Heidenreich 1983). 
2012b). Building on work that integrated disk dynamics and meteorite compositions, Gounelle et al. (2006) estimated the orbit for carbonaceous chondrite Orgueil (Ivuna-type; CI) as similar to the orbits of Jupiter-family comets, and suggested a cometary origin for CI meteorites, as had others before (Lodders and Osborne 1999 and references therein). Considering the uncertainties in the input data (150-year-old visual observations) for the orbital calculations, Gounelle et al. (2006) concluded this was more of a best-informed guess than a certainty; however, there are additional arguments for a connection of CI and CM (Mighei- like; CM) chondrites to comets and asteroids that can be regarded as "extinct comets" (see Lodders and Osborne 1999).

The general placement of where planets and meteorite parent bodies accreted in the disk may be deduced from the 'classical' interpretation of a solar nebula temperature-pressure structure together with the thermal stability of condensed phases from thermodynamic calculations (see Lewis 1974). Most subsequent models also invoked radial gradients in temperature and composition in the disk influencing the composition of the forming planetesimals as functions of location and time. The role dynamical re-distribution of accreted objects played, however, has recently been recognized and complicated the classical interpretation. Numerical models, such as the low-mass asteroid belt, Grand Tack, and early instability models (for review, see Raymond et al. 2020) indicate that the rapid growth and/or gas- driven migration of the giant planets destabilized the orbits of nearby planetesimals, many of which were scattered inward and outwards. This produced an inner asteroid belt dominated by inner solar system objects and an outer belt dominated by outer solar system objects (e.g., Walsh et al. 2011; Raymond and Izidoro 2017). A consequence of dynamical redistribution is that the heliocentric distance at which a planetesimal resides (now) does not necessarily reflect its initial accretion location in the disk.

\subsection{Relating Nucleosynthetic Isotope Signatures of Bulk Meteorites to AccretionLocations in the Disk: The NC-CC Isotope Dichotomy}

In 2011, a dichotomy was identified among solar system objects based on their nucleosynthetic isotope composition. Building on work by Trinquier et al. (2007, 2009), Warren (2011) compiled Ti, Ni, Cr, and O bulk rock isotope data and observed that carbonaceous chondrites were generally more isotopically anomalous than other chondrites (i.e., ordinary and enstatite chondrites), achondrites, Earth, Moon, and Mars. He termed the carbonaceous chondrite group "carbonaceous chondrite, $\mathrm{CC}$ " and the other group "non-carbonaceous chondrite, NC" (Fig. 2). The isotopic bimodality has since been found in siderophile element isotope compositions (e.g., $\mathrm{Mo}, \mathrm{Ru}, \mathrm{W}$ ) of magmatic and non-magmatic iron meteorites (e.g., Budde et al. 2016; Kruijer et al. 2017; Poole et al. 2017; Worsham et al. 2017; Bermingham et al. 2018b). The use of carbonaceous chondrite (CC) and noncarbonaceous chondrite ( $\mathrm{NC}$ ) to describe iron meteorites is a misnomer because iron meteorites are not chondritic meteorites. It has, however, become convention to use the NC-CC notation to describe the isotopic bimodality regardless of whether or not a meteorite is a chondrite as defined by its mineralogy and petrogenesis.

As the $\mathrm{CC}$ and $\mathrm{NC}$ groups do not overlap in nucleosynthetic isotope compositions, the dichotomy appears to have a bimodal origin. Warren (2011) proposed a spatial or temporalorigin for the dichotomy based on the inferred link between the chemical composition of a given parent body and its general accretion location in the protoplanetary disk (following reasoning by Wood 2005; Gounelle et al. 2006; Gounelle et al. 2008). Warren (2011) speculated that all chondritic CC meteorites formed in the outer (relatively volatile-rich) solar system, whereas the NC parent bodies formed in the inner (relatively volatile-depleted) solar system with the two regions possibly being separated by proto-Jupiter.

What the NC and CC isotopic dichotomy represents.2is debated. Kruijer et al. (2017) advanced the interpretation by Warren (2011) that proto-Jupiter caused a segregation of the inner (NC) and outer (CC) protoplanetary disk. Using Mo and W isotope compositions of bulk chondritic and iron meteorites, Kruijer et al. (2017) proposed that these groups exhibit distinct accretion ages: $\mathrm{NC}(<0.4 \mathrm{Ma}$ after CAI formation) and CC $\left(0.9^{+0.4} \mathrm{Ma}\right.$ after CAI formation). Authors concluded the temporal and isotopic constraints implied that the $\mathrm{NC}$ and $\mathrm{CC}$ reservoirs coexisted in the disk (from $1 \mathrm{Ma}$ until at least 3-4 Ma), but not in the same accretion region. To obtain spatial segregation without inward drift of material, authors proposed the formation of a gap in the disk 
caused by the accretion of proto-Jupiter's core within $1 \mathrm{Ma}$ of CAI formation. Authors conjectured that the cause of the isotopic offset between $\mathrm{CC}$ and $\mathrm{NC}$ was an addition of $r$-process-rich material to the $\mathrm{CC}$ reservoir which did not infiltrate the coexisting, yet spatially separated, $\mathrm{NC}$ reservoir.

Scott et al. (2018) extended the meteorite database and also found that the chemical and isotopic characteristics of CC meteorites could be accounted for by formation of CC parent bodies beyond proto-Jupiter. Authors argued, however, the dichotomy cannot be related to temporal variations in the disk because differentiated bodies that exhibit the NC-CC dichotomy accreted 1-3 Ma before chondrites. Recent ${ }^{182} \mathrm{~W}-$ derived accretion ages reported by Hilton et al. (2019) indicate that some CC parent body accretion ages are not resolved from the accretion ages of NC parent bodies. Brasser and Mojzsis (2020) raised doubts about the role Jupiter played in the separation of the NC and CC reservoirs by finding that the separation event may have been caused by a pressure maximum or a dust trap in the disk near the location at which Jupiter later formed.

\subsection{Implications of the NC-CC Dichotomy on Disk Evolution Models}

Despite the absence of consensus on what caused the isotopic difference between the NC and CC groups, it is a common interpretation that the NC group samples material accreted in the inner disk and the CC group samples material accreted in the outer disk. Using this interpretation, conclusions have been drawn about how the composition of the disk, its condensates, and planets evolved.

Models describing the cause of an isotopic difference between the $\mathrm{NC}$ and $\mathrm{CC}$ regions include the addition of material to the $\mathrm{CC}$ region, but not the $\mathrm{NC}$ region. For example, in Mo isotope compositional space $\left(\mu^{94} \mathrm{Mo} v s . \mu^{95} \mathrm{Mo}\right)$, the $\mathrm{NC}$ and CC groups lie on two parallel or close to parallel lines (e.g., Budde et al. 2016; Worsham et al. 2017; Poole et al.2017; Kruijer et al. 2017; Budde et al. 2019; Yokoyama et al. 2019). The lines could be offset by more $r$-process and/or $p$-process material in the CC region than the NC region (e.g., Budde et al. 2016; Worsham et al. 2017; Poole et al. 2017) or different $s$-process components (Stephan and Davis 2019). Yokoyama et al. (2019) proposed the variation in Mo isotope compositions in CC meteorites is related to either local parent-body processing or a heterogeneous distribution of $s$-process matter in the outer solar system. In the $\mathrm{NC}$ group, these authors identified two end-member components (NC-A and NC-B), where the NC-B component reflects the remaining complement of the type B CAIs gaseous source reservoir.

Desch et al. (2018) developed a numerical model explaining why carbonaceous chondrites have an abundance of CAIs (refractory component), which are long-known hosts for nucleosynthetic isotope anomalies. Their model was able to predict where in the disk different meteorite parent bodies formed. Authors found CC and NC bodies formed in the outer and inner disk, respectively, where regions were separated by proto-Jupiter, and that inside Jupiter's orbit, CAIs were depleted by aerodynamic drag. Alexander (2019a, 2019b)performed a comprehensive study of the elemental and isotopic characteristics of CC and NC meteorites. Alexander (2019a) showed that different mixtures of the same four meteorite components can reproduce most bulk elemental and isotopic compositions of $\mathrm{CC}$ meteorites, but $\mathrm{NC}$ meteorites require distinctly different mixtures (Alexander 2019b). Burkhardt et al. (2019) found that by mixing "CAI-like" material into an NC-like composition, the chemical and isotopic composition of the $\mathrm{CC}$ reservoir could be produced.

Nanne et al. (2019) and Burkhardt et al. (2019) proposed similar models detailing the cause of distinct isotope compositions in CAIs, the NC, and CC groups. A variant of the infall model was published by Spitzer et al. (2020). Authors propose that the most straight-forward explanation for a change in isotope composition (between $\mathrm{NC}$ and $\mathrm{CC}$ ) is a change in the composition of infalling material from the parental molecular cloud into the disk, cou- pled with variable mixing and subsequent isolation (by proto-Jupiter) of reservoirs within the disk (e.g., Nanne et al. 2019).

As an example, the model by Nanne et al. (2019) can be distilled into the following: (1) early infall into the disk occurred where molecular cloud material enriched in presolar carriers produced in neutron-rich environments (e.g., ${ }^{58} \mathrm{Ni},{ }^{50} \mathrm{Ti},{ }^{54} \mathrm{Cr}$ and $r$-process Mo) was added to the disk; (2) CAIs, which formed first and close to the Sun, inherited this mix of carriers and are the closest compositional match to the initial isotopic composition of the disk; (3) CAIs were transported outwards by rapid radial expansion of the early infalling material; (4) after removal of the early-formed CAIs, the composition of infalling material changed to be more NC-like, which was not enriched in the neutron-rich nuclides, and thisperiod of infall provided most of the mass 
of the inner disk and it did not reach the outer disk via infall. This scenario produces a dominance of an NC-like composition in the inner solar system, where presumably the NC parent bodies accreted. The outer solar system became more CC-like as a result of inner and outer disk mixing between the initial disk material with the later accreting NC-like material. To avoid homogenization of the disk, the model invokes an early formed protoJupiter that inhibited exchange of material between the inner and outer disk.

Although infall models have appealing features, their feasibility depends on central assumptions that are yet to be verified. For example, these models rely on a change in the isotopic composition of infalling material. Nanne et al. (2019, pp. 50 and 52, respectively) surmised the change in isotopic composition of infalling material occurred because it came "from different parts of a compositionally heterogeneous parental molecular cloud, or the composition of the cloud itself changed over time" where the "isotopic heterogeneity within the solar system's parental molecular cloud has been maintained within the solar accretion disk because material infalling at different times derives from different parts of the parent cloud". The key questions are what caused the specific change in isotopic composition of the infalling molecular cloud material and over what time period could these changes occur? It was thought that molecular clouds in star forming regions were chemically homoge- neous because star clusters (which formed from large molecular clouds) are homogeneous (e.g., Klessen 2011; Bland-Hawthorn and Freeman 2014). It has also been proposed, how- ever, that chemical heterogeneities (e.g., carbon monoxide and water) may persist between the pre-stellar core, the protostar, and circumstellar disk (Visser et al. 2009). Whether or not this chemical homogeneity extends to the specific presolar carriers of nucleosynthetic isotope anomalies is yet to be verified.

After the onset of star formation in a large molecular cloud, contamination of a presolarmolecular cloud can occur by supernova ejecta (e.g., Bland-Hawthorn and Freeman 2014). The fast evolution of supernovae in star-forming regions is the basis for the supernova- triggered formation of the Sun, including injection of supernova material into the presolar cloud (e.g., Cameron and Truran 1977; Foster and Boss 1997; Boss 2013), or for hypotheses involving later injection of "fresh" supernova material into the evolving solar nebula (e.g., Chevalier 2000; Hester et al. 2004). The injection of supernova material may provide a large proportion of neutron-rich nuclides and nuclides made by the $r$-process. To account for the relatively high ${ }^{26} \mathrm{Al}$ and low ${ }^{60} \mathrm{Fe}$ abundances inferred for the early solar system, however, formation of the solar system may have been initiated by a triggered star formation at the edge of a Wolf-Rayet bubble (Dwarkadas et al. 2017). Nanne et al. (2019), however, concludes that the NC-CC isotope dichotomy reflects isotopic heterogeneity within the solar system's parental molecular cloud that cannot be linked to a specific event around $t_{0}$.

Additionally, the infall models involve CAI formation close to the Sun and subsequent transportation outward by rapid radial expansion of early-infalling material, a similar pro- cess through which the underlying disk was produced (Yang and Ciesla 2012; Nanne et al.2019). Early in disk evolution, rapid radial expansion may occur as mass from the infalling cloud is added preferentially to the inner regions of the disk, thereby fueling outward flow (Yang and Ciesla 2012). In Yang and Ciesla (2012), the standard case scenario has infall ending at $\sim 0.3 \mathrm{Ma}$. How the timing and extent of mixing predicted by infall models and rapid radial expansion align with the age and composition constraints of $\mathrm{NC}-\mathrm{CC}$ meteorites requires further consideration. Also needing more detailed assessment is how rapid radial expansion fits with observations and other models of disk formation (e.g., Williams and Cieza 2011). Such models often describe the presolar cloud material accreting onto the protosun and the disk, and the Sun's growth eventually becoming dominated by accretion of disk material. In these models, during the Sun's growth, the accretion flow is from outside- in, and the disk loses mass via accretion onto the star. Further, the effects of outflow and jet formation from the protosun are yet to be fully considered. Here, it is briefly noted that theX-wind models by Shu (1997), Shu et al. (2001) and Lee et al. (1998) are relevant to infallmodels (Yang and Ciesla 2012). From Yang and Ciesla (2012), unresolved issues include the role of magnetic fields, supersonic turbulence, rotation in the cloud, and a variable infall rate. X-wind models (or variants thereof) could provide mechanisms for CAI formation near the Sun, and their transport out to larger heliocentric distances. At this stage, the timing and process of infall and transportation of disk material (including CAIs) to the outer disk needs further investigation.

Central to determining the feasibility of infall models will be constraining the cause and timing of the change in isotopic composition and physical location of the infalling molecular cloud material to the disk, and 
the distribution of disk material in the broader context of what is known about solar nebula evolution. Although important strides are being made, a comprehensive model describing what the NC-CC dichotomy represents, if it reflects in-ner vs. outer solar system compositions, what caused the difference in isotopic composition between and within the two groups, and defining Jupiter's role are topics still under development.

\section{Does the NC-CC Dichotomy Extend to Major Highly-VolatileElements?}

In addition to the implications the NC-CC dichotomy has on protoplanetary disk evolution models, the isotope dichotomy is starting to be used to decipher the source(s) of Earth's water and other volatiles such as C and N. For example, accretion of volatile- rich CC material towards the end of Earth's formation through the Moon-forming impactor and/or late accretion - has been argued for based on a comparison of the $\mathrm{Mo}-\mathrm{Ru}$ isotope composition of meteorites and the bulk silicate Earth (Budde et al. 2019; Hopp et al. 2020). This seems consistent with the constraints provided by elemental com- positions, which require Earth to have accreted from at least two components, initially a reduced one, which now would be sampled by NC meteorites, and later a more oxidized and volatile-rich component, represented by CC meteorites (e.g., Rubie et al. 2015; for a recentreview, see Fegley et al. 2020).

The principle that the NC-CC dichotomy can be used to trace volatile accretion to Earth, however, requires a robust link between the NC-CC isotope signature of meteorite groups, the volatile content of their parent bodies, and the volatile content of the protoplanetary disk from which they accreted. If the NC-CC isotope dichotomy reflects an isotopic heterogeneity between the inner and outer disk, a difference in volatile element abundances may be expected between materials derived from the two reservoirs: non-carbonaceous bodies would be depleted in highly-volatile elements relative to $\mathrm{CC}$ bodies, which are expected to be comparatively volatile-rich. This application of the NC-CC dichotomy is discussed in the sections below which consider the distribution of highly-volatile major elements $(\mathrm{H}, \mathrm{C}$, and $\mathrm{N})$ in the disk and meteorites.

\subsection{Volatile Species and Their Condensation Fronts in the Protoplanetary Disk}

During the protoplanetary disk phase, molecular or atomic volatile species (e.g., $\mathrm{H}_{2}, \mathrm{H}_{2} \mathrm{O}, \mathrm{He}, \mathrm{CO}, \mathrm{CO}_{2}$, $\mathrm{CH}_{4}, \mathrm{CH}_{3} \mathrm{OH}, \mathrm{N}_{2}, \mathrm{NH}_{3}, \mathrm{HCN}, \mathrm{H}_{2} \mathrm{~S}$, etc.) with low condensation temperatures ( $160 \mathrm{~K}$; e.g., Zhang et al. 2015) rested in the gas phase throughout a significant portion of the disk. At sufficiently low temperatures (i.e., below the condensation temper- ature of water), the solid mass density in the disk increased substantially because of water ice formation, thereby aiding planet accumulation. Condensation of other C- and N-bearing ices could have increased the mass density further. In addition, volatile ices likely played an essential role in planet formation by promoting coagulation of 'sticky' ice-coated dust grains into larger pebbles (Wang et al. 2005). Thus, the initial distribution and physical state of volatile species (solid vs. vapor) in the disk was critical in determining the composition and evolution of planetesimals.

To the first order, the thermal structure of the protoplanetary disk controlled where hydrogenated molecules (e.g., $\mathrm{H}_{2} \mathrm{O}$ ) and other volatile compounds (e.g., carbon- and nitrogen- bearing species) condensed into ice or froze out onto small dust grains that were subsequently incorporated into accreting planetesimals. While $\mathrm{H}_{2}$ and $\mathrm{He}$ remained in the gaseous state throughout the disk, water molecules were able to freeze out into solid ice grains at and beyond the so-called "snow line" (i.e., the $\mathrm{H}_{2} \mathrm{O}$ condensation front at $160 \mathrm{~K}$ at the mid- plane; Hayashi 1981; Lodders 2003, 2004; Krijt et al. 2016) (Fig. 4). Other condensation fronts, defined by the physical state of $\mathrm{H}-, \mathrm{C}-$, and N-bearing molecules, included those of ammonia, carbon monoxide, carbon dioxide, methane, methanol, and hydrogen cyanide. Importantly, ice formation depends on the kinetics of the reaction(s) making the condensing molecules. For example, the very stable molecules $\mathrm{CO}$ and $\mathrm{N}_{2}$ essentially contain all the carbon and nitrogen in the solar gas at high temperatures. Only at lower temperatures and high total pressures, will the reaction equilibrium shift to methane and ammonia, i.e., $\mathrm{CO}+3 \mathrm{H}_{2}=\mathrm{CH}_{4}+\mathrm{H}_{2} \mathrm{O}$ and $\mathrm{N}_{2}$ $+3 \mathrm{H}_{2}=2 \mathrm{NH}_{3}$ (Lewis and Prinn 1980; Fegley and Prinn 1989; Fegley 2000; Lodders 2003). However, at the low total pressure within the outer protoplanetary disk, the efficient conversion of $\mathrm{CO}$ to $\mathrm{CH}_{4}$ and of $\mathrm{N}_{2}$ to $\mathrm{NH}_{3}$ is kinetically inhibited. 


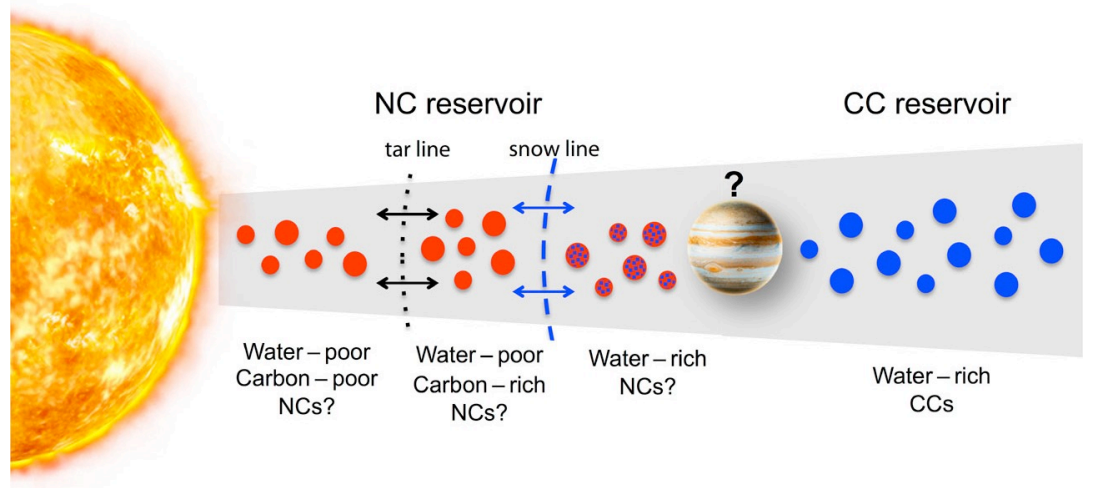

Fig. 4 Schematic cross-section of the protoplanetary disk (not to scale). Planetesimals with NC-and CC-type nucleosynthetic compositions are represented in red and blue, respectively. The two reservoirs are considered to have been spatially separated (e.g., Warren 2011), possibly as a result of the early accretion of proto-Jupiter (Kruijer et al. 2017). Based on the radial gradient in temperature, planetesimals that accreted beyond the snow line (where $T 160 \mathrm{~K}$ at the midplane; Hayashi 1981; Lodders 2004) are expected to be water-rich, whereas 'dry' (i.e., ice-free) planetesimals that formed between the tar- and snow line could have incorporated refractory organics (Lodders 2004; Nakano et al. 2020). The radial position of the mobile condensation fronts relative to the Jupiter barrier likely played a fundamental role in the distribution and transport of inorganic ices (and organics) in the disk during the first few million years of solar system history.

Note that this also affects the total amount of water because $\mathrm{O}$ is locked up in CO. Given that other potential $\mathrm{C}$ - and N-bearing molecules are affected in a similar manner, mod- els of their condensation locations must consider kinetics and dynamical modelling, and it follows that the $\mathrm{NH}_{3}, \mathrm{CH}_{3} \mathrm{OH}$, and $\mathrm{H}_{2} \mathrm{O}$ ice lines essentially coincided while $\mathrm{CO}$ and $\mathrm{CH}_{4}$ condensed at much greater heliocentric distances (e.g., DodsonRobinson et al. 2009; Bergin and Cleeves 2018).

In contrast to these inorganic volatile ices that only exist at $T \leq 160 \mathrm{~K}$, laboratory heat-ing experiments under high vacuum demonstrate that interstellar organic analogs persist at temperatures above 350 to $450 \mathrm{~K}$ (Nakano et al. 2003). Lodders (2004), therefore, argued that the disk also had a "tar line" inward of the snow line, delineating a region where refrac- tory organics (such as poly-aromatic hydrocarbons) were stable but water existed in vapor form only (Fig. 4). Because innermost disk temperatures were above the condensation tem- perature of all volatile ices, planetary objects that formed sunward of the snow line are not expected to have accreted icy grains, in contrast to bodies formed at greater heliocentric distances. Between the snow and tar lines, however, organics, especially their refractory fractions, could have been incorporated into 'ice-free' planetesimals (Fig. 4); these organics represent a potential source of water for bodies accreted sunward of the snow line (Nakanoet al. 2020).

It is important to note that a condensation front is not a straight "line", but rather a surface whose position in the disk and vertical-horizontal structure depends on both temperature and (vapor) pressure in the disk, and, therefore, on the disk mass and heating processes within the disk. Meijerink et al. (2009), for example, pointed out that the heliocentric distance of the water condensation front increases with height above and below the disk midplane due to heating of the disk surface by the central star. Vertical diffusive transport of water vapor across the snow line resulted in condensation and subsequent settling of ice particles and ice-coated grains to the midplane, thereby increasing the dust-to-gas ratio along the midplane and promoting particle growth (e.g., Krijt et al. 2016).

The positions of the snow and tar lines at the midplane are essential when contemplating planetesimal formation and accretion of water ice and organics. Not only were these "lines" 3-dimensional structures, but their location changed with time as the disk's temperature, total pressure, and density evolved (e.g., as a function of the luminosity of the central star, surface density, mass accretion rate, opacity; note that 
condensation also feeds into the disk because opacity sources from the gas are removed and dust/ice opacity is generated) (Davis 2005; Garaud and Lin 2007; Oka et al. 2011; Morbidelli et al. 2016; Sato et al. 2016). The absolute positions (and relative positions to accreting planets, including Jupiter) and rate of change of these positions during the disk phase, however, are model dependent. Different models place the snow line anywhere between $\sim 1$ (e.g., Sasselov and Lecar 2000) and 4 to 7 AU (Cameron 1995; Boss 1996; DodsonRobinson et al. 2009; see Fegley 2000 for a summary of models of $P$ - $T$ conditions in the solar nebula). According to the disk model of Boss (1996), the snow line nevermigrated closer than 3 AU of the Sun. In the model of Garaud and Lin (2007), the snow line migrated to $\leq 1$ AU as the mass accretion rate onto the Sun decreased within the first few million years of the nascent solar system, implying that water ice could condense at $1 \mathrm{AU}$ from the residual nebula gas and that water ice-rich planetesimals existed in the terrestrial planet-forming region. However, Morbidelli et al. (2016) presented a speculative argument that even as the temperature decreased below the water condensation threshold, the disk inwards of Jupiter may have remained ice-depleted because proto-Jupiter effectively prevented the inward drift of icy particles once it reached 20 Earth masses; as a result, the snow line could have "fossilized" at $\sim 3$ AU. This model implies that planetesimals which formed sunward of Jupiter's orbit would not have accreted any water ice. Evidence from NC chondrites and achondrites, however, contradicts this scenario (see Sect. 5.2).

Overall, based on predictions of a strong heliocentric radial gradient in temperature and total pressure, the volatile content and composition of different planetesimals should have been characterized by sharp transitions between species that were refractory enough to freeze out, and those that were too volatile and remained in the gas phase. Planetesimals that accreted close to the Sun are expected to be water- and carbonpoor, whereas bodies that formed in the outer solar system should have accreted H-C-N-rich ices and organics (Fig. 4). The 3-dimensional distribution of ices and vapor, however, continuously evolved over the lifetime of the protoplanetary disk as the condensation fronts moved inwards and outwards. Currently, it is unresolved how this movement affected the distribution of volatile ices in the feeding zone of planetesimals during the first several Ma of solar system his- tory. In addition, there are factors other than temperature and total pressure that control the volatile characteristics of planetesimals and meteorites (see Sect. 5.2). Thus, linking the volatile content of a meteorite to a (radial) accretion location of a planetary body is not straightforward.

\subsection{Major Volatile Element $(\mathrm{H}, \mathrm{C}, \mathrm{N})$ Abundances in $\mathrm{NC}$ and $\mathrm{CC}$ Chondrites}

The distinct nucleosynthetic isotopic composition of $\mathrm{NC}$ - and CC-type meteorites has been interpreted to indicate that their parent bodies accreted in the inner and outer solar system, respectively, separated by Jupiter (Fig. 4). If so, $\mathrm{NC}$ and $\mathrm{CC}$ meteorites could serve (to the first order) as probes of the volatile element signatures of different reservoirs. Do the volatile contents of these objects follow the expected trend that NC meteorites are more volatile-poor than $\mathrm{CC}$ meteorites?

The H-C-N content of achondrites (and presumably larger differentiated planetary building blocks) does not reflect that of the disk or of undifferentiated precursors because melting and differentiation caused decomposition of H-C-N carrier phases (such as organics), variable degrees of degassing, and re-distribution of hydrogen, carbon, and nitrogen between the silicate and metal portions of the parent body (e.g., Mikhail and Füri 2019). Similarly, iron meteorites do not preserve a direct record of the major volatile element abundances of their parent bodies and formation regions because the $\mathrm{H}-\mathrm{C}-\mathrm{N}$ con- tent of planetary cores depends on a complex set of parameters, such as the composition of the Fe-rich alloy (including the abundance of $\mathrm{H}, \mathrm{C}, \mathrm{N}$, $\mathrm{S}$, and $\mathrm{Si}$ ), pressure, temperature, and oxygen fugacity during metal-silicate equilibration (e.g., Wood et al. 2013).

In contrast, the preservation of $\mathrm{H}-\mathrm{C}-\mathrm{N}$ in chondrites indicates that these materials may, to an extent, record the (minimum) volatile content of their parent body and presumably the inherited disk material. Although the volatile content of primitive (i.e., those that are least affected by aqueous alteration or thermal metamorphism and are unshocked) planetesimals is fundamentally controlled by several parameters, such as the timing of accretion, location of accretion relative to the position of the ice lines and tar line, redox conditions, 
as well as the distribution of $\mathrm{H}-\mathrm{C}-\mathrm{N}$ carriers (e.g., presolar grains, organics) in the disk, the H-C- N abundances in different chondrites could reflect the volatile element composition of the region of the disk from which they accreted. Accordingly, can the NC-CC dichotomy be extended to volatile element abundances in chondrites?

Considering examples of NC-type objects, enstatite chondrites are highly reduced, im- plying that their parent bodies formed in an oxygen-poor environment. They have been presumed to be devoid of water (Mason 1966; Hutson and Ruzicka 2000; Rubin and Ma 2017), until Piani et al. (2020) recently showed that enstatite chondrites contain up to 0.06 wt.\% $\mathrm{H}$ (i.e., 0.54 wt.\% equivalent $\mathrm{H}_{2} \mathrm{O}$ ). Ordinary chondrites contain slightly larger quantities of water (i.e., $\leq 0.2$ wt.\% H; Alexander et al. 2012, 2018; Vacher et al. 2020; Fig. 3), indicating that their parent bodies, which formed $\sim 2$ Ma after CAIs at 2 AU (e.g., Henke et al. 2012; Blackburn et al. 2017; Sugiura and Fujiya 2014), accreted some water (ice). Sutton et al. (2017) argued that a significant amount (i.e., up to $\sim 80 \%$ ) of water was lost on the parent body of Semarkona, the most primitive ordinary chondrite known, as a result of Fe oxidation by water during aqueous alteration and/or metamorphic dehydration. If large amounts of reactive (liquid) water had been present, much of the iron metal in ordinary chondrites would have been converted to magnetite, and sulfides would have been attacked; this, how- ever, is not the case. Nonetheless, the hydrogen content of ordinary chondrites, together with the presence of hydrated minerals (smectite) and secondary phases (calcite) (Alexander et al. 1989), suggests that the temperature had dropped below the condensation temperature of water ice at $\sim 2 \mathrm{AU}$ by the time the ordinary chondrites formed (Alexander et al. 2018). Furthermore, it requires either that some small icy particles and/or organics (inherited, in part, from the interstellar medium and preserved in the outer solar nebula; Lodders 2004; Deloule et al. 1998; Piani et al. 2015) were able to get past Jupiter, or that water-bearing material was trapped sunward of Jupiter's orbit prior to the rapid growth of its core.

Considering CC-type bodies, $\mathrm{CI}$ and $\mathrm{CM}$ carbonaceous chondrites are significantly more water-rich (containing up to 10 to $20 \mathrm{wt. \%}$ equivalent water in the form of hydrous minerals; Kerridge 1985; Pearson et al. 2001; Piani et al. 2020; Robert and Epstein 1982; Robert 2003; Vacher et al. 2020; Fig. 3) ${ }^{6}$, suggesting that their parent bodies formed near or beyond the snow line, where they accreted variable amounts of water-ice grains (Vacher et al. 2020). However, several carbonaceous chondrite groups (e.g., Vigarano-like CV and Karoonda-like CK chondrites) contain only small quantities of water (i.e., $\leq 0.03$ to 0.19 wt.\% H; Vacher et al. 2020; Fig. 3). Although the water abundances of their parent bodies were modified by thermal metamorphism and/or metal oxidation (Sutton et al. 2017; Vacher et al. 2020), these observations indicate that CC-type bodies cannot systematically be classified as "water-rich". Overall, there is no sharp difference in the water mass fraction of planetesimals inferred to have accreted in the inner (ordinary chondrites) and outer solar system (carbonaceous chondrites).

Other volatile element abundances also do not display systematic radial variations. Some members of both the ordinary and enstatite chondrite classes contain more carbon and nitro- gen than certain carbonaceous chondrites (Grady et al. 1986; Wasson and Kallemeyn 1988; Hashizume and Sugiura 1995; Grady and Wright 2003; Rubin and Choi 2009); parent body alteration processes, however, may have significantly affected bulk chondrite carbon and nitrogen abundances (e.g., Pearson et al. 2006). Carbonaceous chondrites themselves record a wide range of carbon $(\leq 0.2$ to $\sim 5 \mathrm{wt} . \%)$ and nitrogen $(\leq 0.01$ to $0.5 \mathrm{wt} . \%)$ abundances (e.g., Pearson et al. 2006, and references therein) (Fig. 3). Some (ppm-levels) of the carbon and nitrogen occurs in the form of presolar grains (see Sect. 2) but most is present as organic matter dispersed in the matrix of carbonaceous chondrites, and in the form of reduced species (graphite, carbides, nitrides) in enstatite chondrites (e.g., Grady and Wright 2003). This suggests that the accretion and retention of nominally volatile elements (C, N) in the inner regions of the disk may have been facilitated by different oxidation states. Before this can be concluded, however, details about the evolution of the redox conditions in the disk, including how they affected chemical reactions in the nebula and the dynamics of accretion, need to be further documented.

The key point is that planetesimals which formed in the NC reservoir cannot be generally characterized as "volatile-depleted" with respect to volatile and highly volatile elements compared to CC bodies. Although 
they are relatively deficient in water and carbon as compared to some bodies accreted within the CC reservoir, several volatile elements are comparable in abundance in the most reduced (enstatite chondrites from the NC group) and oxidized (the carbonaceous chondrites from the CC group) chondrites. Thus, with current datasets, the concept of the NC-CC dichotomy cannot yet be extended to all volatile element abundances, and explanations are needed to account for the discrepancies between bulk volatile contents and the NC-CC grouping of meteorites. In addressing these discrepancies, it will be important to determine the extent to which the volatile element composition of a meteorite reflects that of its parent body and the disk region the parent body sampled.

\section{Implications for Volatile Accretion: Building Earth from NC- andCC-Type Precursors}

Based on oxygen and nucleosynthetic isotope compositions, Earth is classified as a NC- body (Warren 2011; Fig. 2), although in $\mu^{94}$ Mo vs. $\mu^{95}$ Mo compositional space Earth lies between the NC and CC groups (Budde et al. 2019). This classification implies that early Earth's building blocks were dominated by materials with NC isotopic signatures. Although it has been proposed that in elemental composition Earth is relatively close to carbonaceous chondrites, in particular CV and CK chondrites (e.g., Palme and O'Neill 2003), oxygen isotopes and numerous nucleosynthetic isotope data preclude more than a few percent carbonaceous chondrite material accreting to Earth. These data require that Earth formed from a reservoir that is isotopically similar to enstatite chondrites (or a mixture of bodies that bracket the isotopic composition of Earth) (e.g., Clayton et al. 1984; Lodders 2000; Dauphas 2017). Earth cannot have been constructed solely out of enstatite chondrites because of the resolvable chemical (including oxidation state) and mass-dependent isotopic differences between enstatite chondrites and Earth (e.g., McDonough and Sun 1995; Lodders 2000; Drake and Righter 2002; Fegley et al. 2020). Dauphas (2017) proposed that enstatite meteorites and Earth formed from the same isotopic nebular reservoir, and the reason that the bodies differ in bulk chemical compositions is because their chemical evolution diverged due to fractionation via nebular and planetary processes.

Determining the role that NC- and CC-type bodies (and chondrites vs. achondrites) played in the accretionary history of Earth can become complicated when the isotopic and chemical characteristics of an object might be decoupled. Given the absence of meteoritic materials that match Earth's elemental and isotopic compositions, it is likely that Earth was primarily constructed out of building blocks that shared feeding zones with enstatite chondrites, but these materials are not sampled by the current meteorite collection (e.g., Drake and Righter 2002; Dauphas 2017; for a recent review, see Fegley et al. 2020).

\subsection{Constraints from Hydrogen Isotopes}

The present-day Earth has a significant quantity of water at its surface, and five to ten ocean masses worth of hydrogen as $\mathrm{OH}$ and $\mathrm{H}_{2} \mathrm{O}$ may reside in the terrestrial mantle (e.g., Marty 2012; Marty et al. 2016; Hirschmann 2018). The source of Earth's water, and the timing of water accretion, is an ongoing investigation in planetary science, with models proposing different timing and means via which water was delivered to Earth (e.g., via nebular ingassing, asteroids, or comets; e.g., Marty 2012; Hallis et al. 2015; Marty et al. 2016; Alexander 2017; Alexander et al. 2018; Wu et al. 2018). Notably, the recent detection of significant quantities of water (i.e., up to $0.54 \mathrm{wt} . \%$ equivalent $\mathrm{H}_{2} \mathrm{O}$ ) in enstatite chondrites represents a paradigm shift in the understanding of the origin of Earth's water, as a significant, even dominant fraction of terrestrial water could be derived from EC-like bodies (Piani et al. 2020).

\footnotetext{
${ }^{6}$ Measurable retention of any originally accreted water (ice) on the parent body requires post-accretion aqueous alteration; the water abundance then also depends on kinetics and the degree of aqueous processing and/or thermal metamorphism on the parent body. It should be noted that important intra- and inter-chondrite variations in water concentrations exist which can be explained, in part, by varying proportions of H-bearing components in a given (sub-)sample (e.g., Pearson et al. 2001). Analytically, not all extraction techniques may clearly distinguish between primordial and (terrestrial) adsorbed water (e.g., Robert and Epstein 1982; Vacher et al. 2020), and terrestrial exposure of "finds" can modify water concentrations (Stephant et al. 2018). Thus, the reported bulk water contents of chondrites cannot directly reflect the original accreted water in allcases.
} 
Whether or not planetesimals from the inner or outer solar system were a dominant source of Earth's water can be further interrogated by comparing the $\mathrm{H}$ isotope composition of meteorites with the terrestrial mantle. Hydrogen isotopes ( $\delta \mathrm{D}=-218$ to $-125 \%$ in the deep Earth's mantle; Deloule et al. 1991; Hallis et al. $2015)$ identify enstatite-type objects $\left(\delta \mathrm{D}_{\mathrm{EC} \text {,bulk }}=-146\right.$ to $-98 \%$; Piani et al. 2020$)$ or carbonaceous $(\mathrm{CI} / \mathrm{CM})$ chondrite- like material $\left(\delta \mathrm{D}_{\mathrm{C} / \mathrm{CM}}=-227\right.$ to $+300 \%$; e.g., Alexander et al. 2012; Marty et al. 2016; Alexander et al. 2018; Piani et al. 2020), ${ }^{7}$ as the potential sources of Earth's water, with minor $(<1 \%)$ cometary (Marty et al. 2016) and nebular (Hallis et al. 2015; Wu et al. 2018) contributions. A nebular volatile contribution is independently indicated by the presence of solar-like neon in Earth's mantle (Honda et al. 1993; Yokochi and Marty 2004; Williams and Muhkopadyay 2018), but mass balance considerations suggest that this contribution was minor for major volatiles such as hydrogen, carbon, and nitrogen (e.g., Marty 2012).

The new findings of Piani et al. (2020) identify EC-type bodies as the likely sources of water in Earth's interior. However, Earth's surficial (oceans and atmosphere) water reservoir, which is more enriched in D than the mantle, appears to require a contribution of carbonaceous chondrite-like material (Piani et al. 2020). The most likely explanation for the accretion of this component is that 'wet' (i.e., 'icy') CI/CM-type bodies were scattered inward from greater heliocentric distances as a result of Jupiter's growth and/or gas-driven migration after the first 3 to 4 million years of solar system history (Raymond and Izidoro 2017; Alexander et al. 2018).

\subsection{Constraints from Siderophile Element Isotopes}

The importance of late accretion (often referred to as the "late veneer") for adding water to Earth remains a matter of debate. Late accretion provided the highly siderophile elements to Earth's mantle after core formation (Chou 1978) and represents a contribution of $\sim 0.5 \mathrm{wt} \%$ (by mass) of chondritic material (Walker 2009). Based on highly siderophile element isotope signatures, this material was inferred to originate from the in- ner solar system, which may imply that late accretion did not provide a significant amount of water to our planet (Bermingham and Walker 2017; Fischer-Gödde and Kleine 2017; Bermingham et al. 2018b). A recent comparison of the $\mathrm{Ru}$ isotope composition of Archeanrocks from South West Greenland (Fischer-Gödde et al. 2020) with the modern silicate mantle (Bermingham and Walker 2017), however, indicates that carbonaceouschondrite- like material may have been delivered to Earth during late accretion. The implications for the origin of Earth's water are yet to be determined because the extent to which the unusual Ru isotope composition of South West Greenland represents the mantle composition before late accretion is as yet unknown (Bermingham 2020). Future work that determines the extent of Ru isotope variability in Earth's mantle will be important for constraining the origin and timing of delivery of Earth's volatiles.

\subsection{Constraints from Nitrogen Isotopes}

A clear link between nitrogen abundance and the NC-CC classification is yet to be determined (see Sect. 5.2). However, a dichotomy seems to exist for nitrogen isotope $\left({ }^{15} \mathrm{~N} /{ }^{14} \mathrm{~N}\right)$ ratios, with $C C$ irons being enriched in ${ }^{15} \mathrm{~N}$ compared to $\mathrm{NC}$ irons (Scott et al. 2018). These observations are consistent with the general trend of increasing ${ }^{15} \mathrm{~N}$-enrichments with radial distance from the Sun (Füri and Marty 2015), i.e., from the Sun $\left(\delta^{15} \mathrm{~N}_{\text {Sun }}=-383 \pm 8 \%\right.$; Marty et al. 2011) to the Earth-Moon system $\left(\delta^{15} \mathrm{~N}_{\text {Earth-Moon }} \approx-40\right.$ to $+26 \%$; Palotet al. 2012; Cartigny and Marty 2013; Füri et al. 2015; Labidi et al. 2020) and Mars $\left(\delta^{15} \mathrm{~N}_{\text {Mars,mantle }}\right.$ $-30 \%$; Mathew and Marti 2001) and to comets $\left(\delta^{15} \mathrm{~N}_{\text {Comet }} \approx+800\right.$ to $1000 \%$; Bockelée-Morvan et al. 2015, and references therein). In contrast, nitrogen isotope ratios recorded by chondrites do not display the NC-CC dichotomy. Enstatite chondrites are characterized by low $\delta^{15} \mathrm{~N}$ values $\left(\delta^{15} \mathrm{~N}_{\mathrm{EC}}=-47\right.$ to $0 \%$; Kung and Clayton 1978; Grady et al. 1986), but the nitrogen isotopic compositions of ordinary $\left(\delta^{15} \mathrm{~N}_{\mathrm{OC}}=-4\right.$ to $+75 \%$;

\footnotetext{
${ }^{7}$ Individual $\mathrm{CI}$ and $\mathrm{CM}$ chondrites record a wide range of hydrogen isotope $(\delta \mathrm{D})$ values, possibly due, in part, to variable degrees of aqueous alteration and sampling biases (Alexander et al. 2012, 2018; Kerridge 1985; Robert 2003). Therefore, Alexander et al. (2018) used average values of $78 \pm 7 \%$ and $-53 \pm 130 \%$ for the $\mathrm{CI}$ and $\mathrm{CM}$ chondrite groups, respectively.
} 
Hashizume and Sugiura 1995) and carbonaceous chondrites $\left(\delta^{15} \mathrm{~N}_{\mathrm{CI} / \mathrm{CM}}=-6\right.$ to $+56 \%$, excluding Bells (CM2); Kerridge 1985; Alexander et al. 2012) largely overlap. ${ }^{8}$ Since only enstatite chondrites have negative $\delta^{15} \mathrm{~N}$ values, Javoy et al. (1986) suggested that they are the source of nitrogen in Earth's primitive mantle $\left(\delta^{15} \mathrm{~N}\right.$ $\approx-40 \%$; Palot et al. 2012; Cartigny and Marty 2013; Dalou et al. 2019). As outlined in Sect. 5.2, enstatite chondrites contain a small but significant amount of water, and they contain important quantities of nitrogen, possibly as a result of enhanced nitrogen solubility under the reducing conditions at which their parent bodies formed (Libourel et al. 2003; Boulliung et al. 2020). Therefore, enstatite chondrite-like bodies, presumably derived from the NC reservoir (Warren 2011), are not only strong candidates for Earth's primary building blocks but also for the dominant source of terrestrial water and nitrogen (Javoy et al. 1986; Lodders 2000; Dauphas 2017; Piani et al. 2020).

It is noteworthy that the nitrogen isotopic signature of the modern (upper) terrestrial mantle $\left(\delta^{15} \mathrm{~N} \approx-5\right.$ $\pm 2 \%$ in diamonds and mid-ocean ridge basalts; Cartigny and Marty 2013) has previously been interpreted to reflect that $\mathrm{CI} / \mathrm{CM}$-like planetesimals were the major sources of nitrogen on Earth (e.g., Alexander 2017). Yet, Li et al. (2016) and Dalou et al. (2019) recently showed that core formation, possibly coupled with degassing, could have increased the $\delta^{15} \mathrm{~N}_{\text {mantle }}$ value from an enstatite chondrite-like signature to the present-day value. The degree of $\mathrm{N}$ isotope fractionation, however, depends on the redox conditions and on $\mathrm{N}$ speciation. The distinct nitrogen isotope signature of other terrestrial reservoirs such as the atmosphere and crust $\left(\delta^{15} \mathrm{~N} \quad 0\right.$ to $+6 \%$; Cartigny and Marty 2013) suggests that CC-type material contributed some nitrogen to an enstatite chondritelike Earth (Javoyet al. 1986).

Together, Earth's hydrogen, nitrogen, oxygen, and nucleosynthetic isotope signatures of refractory elements such as those shown in Fig. 2 suggest that the terrestrial building blocks may have included both NCand CC-type bodies. A contribution of CC-type (CI- and/or CM-like) material may also be required to explain the isotopic composition of hydrogen and nitrogen in the interiors of Mars and Earth's Moon (Alexander 2017; Füri et al. 2015, 2017). If so, this would imply that the NC and CC reservoirs communicated with each other during the main stages of terrestrial planet accretion. Given the lack of any (known) samples from Venus and Mercury, however, it remains unclear if terrestrial planets closer to the Sun accreted both NC- and CC-type material, and if so, how much of each type.

Challenges, however, remain in relating meteorite volatile compositions to accretion locations in the disk, some of which have been raised in this chapter. These complicate the interpretation that NC meteorites represent inner disk material that is strictly "volatile-poor" relative to $\mathrm{CC}$ meteorites which represent the outer disk and are "volatile-rich"; As such, if and how the NC-CC dichotomy can be used to reconstruct planetary volatile accretion requires further investigation.

\footnotetext{
${ }^{8}$ The different chondrite groups (e.g., CI, CM, CR, CO, CV, CK) within a given class (e.g., carbonaceous chondrites) record a wide range of $\delta^{15} \mathrm{~N}$ values (e.g., Pearson et al. 2006). For a given group, the $\delta^{15} \mathrm{~N}$ value varies with petrologic type, i.e., the extent of thermal metamorphism; $\delta^{15} \mathrm{~N}$ generally decreases with increasing petrologic type from 1 to 4 (Pearson et al. 2006). In addition, the nitrogen extraction method (combustion vs. pyrolysis) can yield distinct $\delta^{15} \mathrm{~N}$ values (e.g., Grady et al. 1986). Therefore, care should be taken when comparing data from different studies.
}

\section{Conclusions}

The NC-CC isotope dichotomy appears to record fundamental chemical and/or physical processes in the disk. Although there is an abundance of models and circumstantial evidence that converge on the idea that the parent bodies of CC meteorites formed in the outer proto- planetary disk beyond Jupiter's orbit and the snow line, irrefutable evidence of their (radial) accretion location is yet to be identified. It remains to be answered what the relationship is between nucleosynthetic isotope signatures in meteorites, their volatile compositions, and their precursor disk composition, and thus if the combination of a nucleosynthetic isotope composition and volatile element content can trace communication between different regions of the disk. Detailed observations of the planet forming process(es) in neighboring systems, synthesized with disk and planetary accretion models and the chemical/isotopic compositions of meteorites, will undoubtedly provide the most comprehensive understanding of the evolution of the protoplanetary disk. These data will be essential to resolving what the NC- 
$\mathrm{CC}$ isotope dichotomy represents. Does the NC-CC isotope dichotomy provide constraints on the temporal, spatial, and chemical evolution of the protoplanetary disk or does it not because of decoupling between the isotopic and chemical characteristics of an object? Resolving this and other issues is important to determining the significance of the NC-CC isotope dichotomy and if the NC-CC character of a parent body permits it to be used to trace the interplay between planetary building blocks.

Acknowledgements We thank the International Space Science Institute (Bern, CH) for hosting and sup- porting the workshop "Reading Terrestrial Planet Evolution in Isotopes and Element Measurements" and the editors of this book. Our sincere thanks to editor in chief H. Lammer for his guidance and patience. We also thank thorough reviews by A.M. Davis and C.M.O'D. Alexander. KRB was supported by NASA Emerging Worlds grants 80NSSC18K0496 and NNX16AN07G, NASA SSERVI grant NNA14AB07A, and the De- partment of Earth and Planetary Sciences, Rutgers University. EF and BM were supported by the European Research Council (ERC) under the European Union's Horizon 2020 research and innovation program (grant agreements no. 715028 and no. 695618, respectively). Work by KL was supported in part by NSF grant AST 1517541 and the McDonnell Centre for the Space Sciences. This is CRPG-CNRS contribution 2374.

\section{References}

C.M.O’D. Alexander, The origin of inner solar system water. Philos. Trans. R. Soc. A, Math. Phys. Eng. Sci. 375, $20150384(2017)$

C.M.O'D. Alexander, Quantitative models for the elemental and isotopic fractionations in chondrites: the carbonaceous chondrites. Geochim. Cosmochim. Acta 254, 277-309 (2019a)

C.M.O'D. Alexander, Quantitative models for the elemental and isotopic fractionations in the chondrites: the non-carbonaceous chondrites. Geochim. Cosmochim. Acta 254, 246-276 (2019b)

C.M.O'D. Alexander, D.J. Barber, R. Hutchison, The microstructure of Semarkona and Bishunpur. Geochim. Cosmochim. Acta 53, 3045-3057 (1989)

C.M.O'D. Alexander, L.R. Nittler, The galactic evolution of Si, Ti, and O isotopic ratios. Astrophys. J. 519,222-235 (1999)

C.M.O'D. Alexander, R. Bowden, M.L. Fogel, K.T. Howard, C.D. Herd, L.R. Nittler, The provenances of asteroids, and their contributions to the volatile inventories of the terrestrial planets. Science 337, 721- 723 (2012)

C.M.O’D. Alexander, K.D. McKeegan, K. Altwegg, Water reservoirs in small planetary bodies: meteorites, asteroids, and comets. Space Sci. Rev. 214, 36 (2018)

S. Amari, L.R. Nittler, E. Zinner, K. Lodders, R.S. Lewis, Presolar SiC grains of Type A and B: Their isotopic compositions and stellar origins. Astrophys. J. 559, 463-483 (2001)

E.A. Bergin, L.I. Cleeves, Chemistry during the gas-rich stage of planet formation, in Handbook of Exoplan- ets, ed. by H. Deeg, J. Belmonte (Springer, Cham, 2018), pp. 2221-2250

K.R. Bermingham, K. Mezger, E.E. Scherer, M.F. Horan, R.W. Carlson, D. Upadhyay, T. Magna, A. Pack, Barium isotope abundances in meteorites and their implications for early Solar System evolution. Geochim. Cosmochim. Acta 175, 282-298 (2016)

K.R. Bermingham, R.J. Walker, The ruthenium isotopic composition of the oceanic mantle. Earth Planet. Sci. Lett. 474, 466-473 (2017)

K.R. Bermingham, N. Gussone, K. Mezger, J. Krause, Origins of mass-dependent and mass-independent Ca isotope variations in meteoritic components and meteorites. Geochim. Cosmochim. Acta 226, 206-223 (2018a)

K.R. Bermingham, E.A. Worsham, R.J. Walker, New insights into Mo and Ru isotope variation in the nebula and terrestrial planet accretionary genetics. Earth Planet. Sci. Lett. 487, 221-229 (2018b)

K.R. Bermingham, Ancient rock bears isotopic fingerprints of Earth's origins. Nature 579, 195-196 (2020)

T. Bernatowicz, G. Fraundorf, T. Ming, E. Anders, B. Wopenka, E. Zinner, P. Fraundorf, Evidence for inter- stellar SiC in the Murray carbonaceous chondrite. Nature 330, 728-730 (1987)

D.C. Black, R.O. Pepin, Trapped neon in meteorites - II. Earth Planet. Sci. Lett. 6, 395-405 (1969)

T. Blackburn, C.M.O'D. Alexander, R. Carlson, L.T. Elkins-Tanton, The accretion and impact history of the ordinary chondrite parent bodies. Geochim. Cosmochim. Acta 200, 201-217 (2017)

J. Bland-Hawthorn, K. Freeman, The Origin of the Galaxy and Local Group, Saas-Fee Advanced Course, vol. 37 (Springer, Berlin, 2014), p. 1

D. Bockelée-Morvan, U. Calmonte, S. Charnley, J. Duprat, C. Engrand, A. Gicquel, M. Hässig, E. Jehin, H. Kawakita, B. Marty, S. Milam, A. Morse, P. Rousellot, S. Sheridan, E.S. Wirström, Cometary isotopes measurements. Space Sci. Rev. 197, 47-83 (2015)

A.P. Boss, Collapse and fragmentation of molecular cloud cores. II. Collapse induced by stellar shock waves. Astrophys. J. 439, 224-236 (1995)

A.P. Boss, Evolution of the solar nebula. III. Protoplanetary disks undergoing mass accretion. Astrophys. J. 469, 906-920 (1996)

A.P. Boss, Mixing and transport of short-lived and stable isotopes and refractory grains in protoplanetary disks. Astrophys. J. 773, 5 (2013)

J. Boulliung, E. Füri, C. Dalou, L. Tissandier, L. Zimmermann, Y. Marrocchi, Oxygen fugacity and melt composition controls on nitrogen solubility in silicate melts. Geochim. Cosmochim. Acta 284, 120-133 (2020)

A.J. Brearley, The action of water, in Meteorites and the Early Solar System II, ed. by D. Lauretta, H.Y. McSween Jr. (University of Arizona Press, Tucson, 2006), pp. 587-624

R. Brasser, S.J. Mojzsis, The partitioning of the inner and outer Solar System by a structured protoplanetary disk. Nat. Astron. 4, 492$499(2020)$ 
G. Budde, C. Burkhardt, G.A. Brennecka, M. Fischer-Gödde, T.S. Kruijer, T. Kleine, Molybdenum iso- topic evidence for the origin of chondrules and a distinct genetic heritage of carbonaceous and non- carbonaceous meteorites. Earth Planet. Sci. Lett. 454, 293-303 (2016)

G. Budde, C. Burkhardt, T. Kleine, Molybdenum isotopic evidence for the late accretion of outer Solar System material to Earth. Nat. Astron. 3, 736-741 (2019)

C. Burkhardt, N. Dauphas, U. Hans, B. Bourdon, T. Kleine, Elemental and isotopic variability in solar system materials by mixing and processing of primordial disk reservoirs. Geochim. Cosmochim. Acta 261, 145-170 (2019)

A.G.W. Cameron, The first ten million years in the solar nebula. Meteoritics 30, 133-161 (1995)

A.G.W. Cameron, W. Truran, The supernova trigger for formation of the solar system. Icarus 30, 447-461 (1977)

P. Cartigny, B. Marty, Nitrogen isotopes and mantle geodynamics: the emergence of life and the atmosphere- crust-mantle connection. Elements 9, 359-366 (2013)

R.A. Chevalier, Young circumstellar disks near evolved massive stars and supernovae. Astrophys. J. 538, L151-L154 (2000)

C.-L. Chou, Fractionation of siderophile elements in the Earth's upper mantle, in Proceedings of the 9th Lunar and Planetary Science Conference (1978), pp. 219-230

L.A. Cieza, S. Casassus, J. Tobin, S.P. Bos, J.P. Williams, S. Perez, Z. Zhu, C. Caceres, H. Canovas, M.M. Dunham, A. Hales, J.L. Prieto, D.A. Principe, M.R. Schreiber, D. Ruiz-Rodriguez, A. Zurlo, Imaging the water snow-line during a protostellar outburst. Nature 535, 258-261 (2016)

R.N. Clayton, L. Grossman, T.K. Mayeda, A component of primitive nuclear composition in carbonaceous meteorites. Science 182, 485-488 (1973)

R.N. Clayton, T.K. Mayeda, A.E. Rubin, Oxygen isotopic compositions of enstatite chondrites and aubrites.J. Geophys. Res. 89, 245249 (1984)

D.D. Clayton, Cosmic chemical memory: a new astronomy. Q. J. R. Astron. Soc. 23, 174-212 (1982)

R.N. Clayton, Solar system: self-shielding in the solar nebula. Nature 415, 860-861 (2002)

E.A. Cloutis, P. Hudon, T. Hiroi, M.J. Gaffey, P. Mann, J.F. Bell III., Spectral reflectance properties of car-bonaceous chondrites: 6. CV chondrites. Icarus 221, 328-358 (2012a)

E.A. Cloutis, P. Hudon, T. Hiroi, M.J. Gaffey, Spectral reflectance properties of carbonaceous chondrites: 7. CK chondrites. Icarus 221, 911-924 (2012b)

J.N. Connelly, M. Bizzarro, Pb-Pb chronometry and the early Solar System. Geochim. Cosmochim. Acta 201, 345-363 (2017)

J.N. Connelly, M. Bizzarro, A.N. Krot, A. Nordlund, D. Wielandt, M.A. Ivanova, The absolute chronology and thermal processing of solids in the solar protoplanetary disk. Science 338, 651-655 (2012)

C. Dalou, E. Füri, C. Deligny, L. Piani, M.-C. Caumon, M. Laumonier, Redox control on nitrogen isotope fractionation during planetary core formation. Proc. Natl. Acad. Sci. USA 116, 11485-14494 (2019)

N. Dauphas, The isotopic nature of the Earth's accreting material through time. Nature 541, 521-524 (2017)

N. Dauphas, E.A. Schauble, Mass fractionation laws, mass-independent effects, and isotopic anomalies. Annu. Rev. Earth Planet. Sci. 44, 709-783 (2016)

A.M. Davis, The cosmochemical history of the pallasites. PhD dissertation, Yale University (1977)

A.M. Davis, J. Zhang, N.D. Greber, J. Hu, F.L.H. Tissot, N. Dauphas, Titanium isotopes and rare Earth patterns in CAIs: evidence for thermal processing and gas-dust decoupling in the protoplanetary disk. Geochim. Cosmochim. Acta 221, 275-295 (2018)

S.S. Davis, Condensation front migration in a protoplanetary nebula. Astrophys. J. 620, 994-1001 (2005)

E. Deloule, F. Albarède, S.M.F. Sheppard, Hydrogen isotope heterogeneities in the mantle from ion probe analysis of amphiboles from ultramafic rocks. Earth Planet. Sci. Lett. 105, 543-553 (1991)

E. Deloule, F. Robert, J.C. Doukhan, Interstellar hydroxyl in meteoritic chondrules: implications for the origin of water in the inner solar system. Geochim. Cosmochim. Acta 62, 3367-3378 (1998)

S.J. Desch, A. Kalyaan, A.C.M. O'D, The effect of Jupiter's formation on the distribution of refractory ele- ments and inclusions in meteorites. Astrophys. J. Suppl. Ser. 238, 11 (2018)

S.E. Dodson-Robinson, K. Willacy, P. Bodenheimer, N.J. Turner, C.A. Beichman, Ice lines, planetesimal composition and solid surface density in the solar nebula. Icarus 200, 672-693 (2009)

M.J. Drake, K. Righter, Determining the composition of the Earth. Nature 416, 39-44 (2002)

V.V. Dwarkadas, N. Dauphas, B. Meyer, P. Boyajian, M. Bojazi, Triggered star formation inside the shell of a Wolf-Rayet bubble as the origin of the solar system. Astrophys. J. 851, 147 (2017)

B. Fegley, Kinetics of gas-grain reactions in the solar nebula. Space Sci. Rev. 92, 177-200 (2000)

B. Fegley, H. Palme, Evidence for oxidizing conditions in the solar nebula from Mo and W depletions in refractory inclusions in carbonaceous chondrites. Earth Planet. Sci. Lett. 72, 311-326 (1985)

B. Fegley, K. Lodders, N.S. Jacobson, Volatile element chemistry during accretion of the Earth. Geochemistry 80, $125594(2020)$

B. Fegley Jr., R.G. Prinn, Solar nebula chemistry: implications for volatiles in the solar system, in The Forma- tion and Evolution of Planetary Systems, ed. by H.A. Weaver, L. Danly (Cambridge University Press, Cambridge, 1989), pp. 171-211

M. Fischer-Gödde, T. Kleine, Ruthenium isotopic evidence for an inner Solar System origin of the late veneer. Nature 541, 525-527 (2017)

M. Fischer-Gödde, B.-M. Magnus, C. Münker, K. Szilas, W.D. Maier, N. Messling, T. Morishita, M. Van Kranendonk, H. Smithies, Ruthenium isotope vestige of Earth's pre-late-veneer mantle preserved in Archaean rocks. Nature 579, 240-244 (2020)

C. Floss, P. Haenecour, Presolar silicate grains: abundance, isotopic and elemental compositions, and the effects of secondary processing. Geochem. J. 50, 3-25 (2016) 
P.N. Foster, A.P. Boss, Injection of radioactive nuclides from the stellar source that triggered the collapse of the presolar nebula. Astrophys. J. 489, 346-357 (1997)

E. Füri, B. Marty, Nitrogen isotope variations in the Solar System. Nat. Geosci. 8, 515-522 (2015)

E. Füri, P.H. Barry, L.A. Taylor, B. Marty, Indigenous nitrogen in the Moon: constraints from coupled nitrogen-noble gas analyses of mare basalts. Earth Planet. Sci. Lett. 431, 195-205 (2015)

E. Füri, E. Deloule, R. Trappitsch, The production rate of cosmogenic deuterium at the Moon's surface. Earth Planet. Sci. Lett. 474, 76-82 (2017)

P. Garaud, D.N.C. Lin, The effect of internal dissipation and surface irradiation on the structure of disks and the location of the snow line around Sun-like stars. Astrophys. J. 654, 606-624 (2007)

S. Gerber, C. Burkhardt, G. Budde, K. Metzler, T. Kleine, Mixing and transport of dust in the early solar nebula as inferred from titanium isotope variations among chondrules. Astrophys. J. Lett. 841, L17 (2017)

M. Gounelle, A. Morbidelli, P.A. Bland, P. Spurný, E.D. Young, M. Sephton, Meteorites from the outer solar system? in The Solar System Beyond Neptune, ed. by M.A. Barucci, H. Boehnhardt, D.P. Cruikshank, A. Morbidelli (University of Arizona Press, Tucson, 2008), pp. 525-541

M. Gounelle, P. Spurný, P.A. Bland, The orbit and atmospheric trajectory of the Orgueil meteorite from historical records. Meteorit. Planet. Sci. 41, 135-150 (2006)

J. Gradie, E. Tedesco, Compositional structure of the asteroid belt. Science 216, 1405-1407 (1982)

M.M. Grady, I.P. Wright, Elemental and isotopic abundances of carbon and nitrogen in meteorite. Space Sci. Rev. 106, 231-248 (2003)

M.M. Grady, I.P. Wright, L.P. Carr, C.T. Pillinger, Compositional differences in enstatite chondrites based on carbon and nitrogen stable isotope measurements. Geochim. Cosmochim. Acta 50, 2799-2813 (1986)

L. Grossman, J.W. Larimer, Early chemical history of the solar system. Rev. Geophys. 12, 71-101 (1974)

F. Gyngard, S. Amari, E. Zinner, K.K. Marhas, Correlated silicon and titanium isotopic compositions of presolar SiC grains from Murchison CM2 chondrite. Geochim. Cosmochim. Acta 221, 154-161 (2018)

L.J. Hallis, G.R. Huss, K. Nagashima, G.J. Taylor, S.A. Halldórsson, D.R. Hilton, M.J. Mottl, K.J. Meech, Evidence for primordial water in Earth's deep mantle. Science 350, 795-797 (2015)

K. Hashizume, N. Sugiura, Nitrogen isotopes in bulk ordinary chondrites. Geochim. Cosmochim. Acta 59, 4057-4069 (1995)

C. Hayashi, Structure of the solar nebula, growth and degay of magnetic fields and effects of magnetic and turbulent viscosities on the nebula. Prog. Theor. Phys. Suppl. 70, 35-53 (1981)

P.R. Heck, J. Greer, L. Kööp, R. Trappitsch, F. Gyngard, H. Busemann, C. Maden, J.N. Ávila, A.M. Davis, R. Wieler, Lifetimes of interstellar dust from cosmic ray exposure ages of presolar silicon carbide. Proc. Natl. Acad. Sci. 1107, 18841889 (2020)

S. Henke, H.-P. Gail, M. Trielof, W.H. Schwarz, T. Kleine, Thermal history modelling of the H chondrite parent body. Astron. Astrophys. 545, A135 (2012)

J.J. Hester, S.J. Desch, K.R. Healy, L.A. Leshin, The cradle of the solar system. Science 304, 1116 (2004)

P.J. Hevey, I.S. Sanders, A model for planetesimal meltdown by ${ }^{26} \mathrm{Al}$ and its implications for meteorite parent bodies. Meteorit. Planet. Sci. 41, 95-106 (2006)

D.C. Hezel, P.A. Bland, H. Palme, E. Jacquet, J. Bigolski, Compositions of chondrules and matrix and theircomplementary relationship in chondrites, in Chondrules: Records of Protoplanetary Disk Processes, ed. by S.S. Russell, H.C. Connolly Jr., A.N. Krot (Cambridge University Press, Cambridge, 2019), pp.91-121

C.D. Hilton, K.R. Bermingham, R.J. Walker, T.J. McCoy, Genetics, crystallization sequence, and age of the South Byron Trio iron meteorites: new insights to carbonaceous chondrite (CC) type parent bodies. Geochim. Cosmochim. Acta 251, 217-228 (2019)

M.M. Hirschmann, Comparative deep Earth volatile cycles: the case for $\mathrm{C}$ recycling from exosphere/ mantle fractionation of major $\left(\mathrm{H}_{2} \mathrm{O}\right.$, $\mathrm{C}, \mathrm{N}$ ) volatiles and from $\mathrm{H}_{2} \mathrm{O} / \mathrm{Ce}, \mathrm{CO}_{2} / \mathrm{Ba}$, and $\mathrm{CO} 2 / \mathrm{Nb}$ exosphere ratios. Earth Planet. Sci. Lett. 502, 262-273 (2018)

N.E. Holden, M.L. Bonardi, P. De Bièvre, P.R. Renne, I.M. andVilla, IUPAC-IUGS common definition and convention on the use of the year as a derived unit of time (IUPAC recommendations 2011). Pure Appl. Chem. 83, 1159-1162 (2011)

M. Honda, I. Mcdougall, D. Patterson, Solar noble gases in the Earth: the systematics of helium-neon isotopes in mantle derived samples. Lithos 30, 257-265 (1993)

T. Hopp, G. Budde, T. Kleine, Heterogeneous accretion of Earth inferred from Mo-Ru isotope systematics. Earth Planet. Sci. Lett. 534, 116065 (2020)

P. Hoppe, S. Amari, E. Zinner, T. Ireland, R.S. Lewis, Carbon, nitrogen, magnesium, silicon, and titanium isotopic compositions of single interstellar silicon carbide grains from the Murchison carbonaceous chondrite. Astrophys. J. 430, 870-890 (1994)

G.R. Huss, J.B. Smith, Titanium isotopic compositions of well-characterized silicon carbide grains from Orgueil (CI): implications for sprocess nucleosynthesis. Meteorit. Planet. Sci. 42, 1055-1075 (2007)

M. Hutson, A. Ruzicka, A multi-step model for the origin of E3 (enstatite) chondrites. Meteorit. Planet. Sci. 35, 601-608 (2000)

T.R. Ireland, E.K. Zinner, S. Amari, Isotopically anomalous Ti in presolar SiC from the Murchison meteorite. Astrophys. J. 376, L53L556 (1991)

M. Javoy, F. Pineau, H. Delorme, Carbon and nitrogen isotopes in the mantle. Chem. Geol. 57, 41-62 (1986)

J.F. Kerridge, Carbon, hydrogen and nitrogen in carbonaceous chondrites: abundances and isotopic composi- tions in bulk samples. Geochim. Cosmochim. Acta 49, 1707-1714 (1985)

R.S. Klessen, in Star Formation in the Local Universe. European Astronomical Society Publications Series, vol. 51 (2011), pp. 133-167

L. Kööp, A.M. Davis, D. Nakashima, C. Park, A.N. Krot, K. Nagashima, T.J. Tenner, P.R. Heck, N.T. Kita, A link between oxygen, calcium and titanium isotopes in ${ }^{26} \mathrm{Al}$-poor hibonite-rich CAIs from Murchison and implications for the heterogeneity of dust reservoirs in the 
solar nebula. Geochim. Cosmochim. Acta 189, 70-95 (2016)

S. Krijt, F.J. Ciesla, E.A. Bergin, Tracing water vapor and ice during dust growth. Astrophys. J. 883, 285 (2016)

A.N. Krot, K. Nagashima, G. Libourel, K.E. Miller, Multiple mechanisms of transient heating events in the protoplanetary disk: evidence from precursors of chondrules and igneous $\mathrm{Ca}$, Al-rich inclusions, in Chondrules: Records of Protoplanetary Disk Processes, ed. by S.S. Russell, H.C. Connolly Jr., A.N. Krot (Cambridge University Press, Cambridge, 2018), pp. 11-56

A.N. Krot, Refractory inclusions in carbonaceous chondrites: records of early solar system processes. Mete-orit. Planet. Sci. 54, 1647$1691(2019)$

T.S. Kruijer, M. Touboul, M. Fischer-Gödde, K.R. Bermingham, R.J. Walker, T. Kleine, Protracted core formation and rapid accretion of protoplanets. Science 344, 1150-1154 (2014)

T.S. Kruijer, C. Burkhardt, G. Budde, T. Kleine, Age of Jupiter inferred from the distinct genetics and forma- tion times of meteorites. Proc. Natl. Acad. Sci. USA 114, 6712-6716 (2017)

T. Kudo, J. Hashimoto, T. Muto, H.B. Liu, R. Dong, Y. Hasegawa, T. Tsukagoshi, M. Konishi, A spatially resolved au-scale inner disk around DM Tau. Astrophys. J. Lett. 868, L5 (2018)

C.C. Kung, R.N. Clayton, Nitrogen abundances and isotopic compositions in stony meteorites. Earth Planet. Sci. Lett. 38, 421-435 (1978)

J. Labidi, P.H. Barry, D.V. Bekaert, M.W. Broadley, B. Marty, T. Giunta, O. Warr, B. Sherwood Lollar,

T.P. Fischer, G. Avice, A. Caracausi, C.J. Ballentine, A. Halldórsson Stefánsson, M.D. Kurz, E.D. Young, Hydrothermal ${ }^{15} \mathrm{~N}^{15} \mathrm{~N}$ abundances constrain the origins of mantle nitrogen. Nature 580, 367-371 (2020)

T. Lee, D.A. Papanastassiou, G.J. Wasserburg, Aluminum-26 in the early solar system: fossil or fuel? Astro- phys. J. 211, L107-110 (1977)

T. Lee, F.H. Shu, S. Shang, A.E. Glassgold, K.E. Rehm, Protostellar cosmic rays and extinct radioactivities in meteorites. Astrophys. J. 506, 898-912 (1998)

J.S. Lewis, The temperature gradient in the solar nebula. Science 186, 440-443 (1974)

J.S. Lewis, R.G. Prinn, Kinetic inhibition of CO and N2 reduction in the solar nebula. Astrophys. J. 238, 357-364 (1980)

R.S. Lewis, T. Ming, J.F. Wacker, E. Anders, E. Steel, Interstellar diamonds in meteorites. Nature 326, 160- 162 (1987)

Y. Li, B. Marty, S. Shcheka, L. Zimmermann, H. Keppler, Nitrogen isotope fractionation during terrestrial core-mantle separation. Geochem. Perspect. Lett. 2, 138-147 (2016)

G. Libourel, B. Marty, F. Humbert, Nitrogen solubility in basaltic melt. Part I. Effect of oxygen fugacity. Geochim. Cosmochim. Acta 67, 4123-4135 (2003)

K. Lodders, An oxygen isotope mixing model for the accretion and composition of rocky planets. Space Sci. Rev. 92, 341-354 (2000)

K. Lodders, Solar system abundances and condensation temperatures of the elements. Astrophys. J. 591, 1220-1247 (2003)

K. Lodders, Jupiter formed with more tar than ice. Astrophys. J. 611, 587-597 (2004)

K. Lodders, S. Amari, Presolar grains from meteorites: remnants from early times of the solar system. Chem. Erde 65, 93-166 (2005)

K. Lodders, B. Fegley, Chemistry of the Solar System (Royal Society of Chemistry, Cambridge, 2011). 476 pp.

K. Lodders, R. Osborne, Perspectives on the comet-asteroid-meteorite link. Space Sci. Rev. 90, 289-297 (1999)

G.J. MacPherson, Calcium-aluminum-rich inclusions in chondritic meteorites, in Meteorites and Cosmo- chemical Processes, vol. 1, ed. by A.M. Davis (Elsevier, Oxford, 2014), pp. 139-179. Treatise on Geo- chemistry, 2nd edn., ed. by H.D. Holland, K.K. Turekian

B. Marty, The origins and concentrations of water, carbon, nitrogen and noble gases on Earth. Earth Planet. Sci. Lett. 313-314, 56-66 (2012)

B. Marty, M. Chaussidon, R.C. Wiens, A.J.G. Jurewicz, D.S. Burnett, A ${ }^{15}$ N-poor isotopic composition for the Solar System as shown by Genesis solar wind samples. Science 332, 1533-1536 (2011)

B. Marty, G. Avice, Y. Sano, K. Altwegg, H. Balsiger, M. Hässig, A. Morbidelli, O. Mousis, M. Rubin, Origins of volatile elements (H, $\mathrm{C}, \mathrm{N}$, noble gases) on Earth and Mars in light of recent results from the ROSETTA cometary mission. Earth Planet. Sci. Lett. 441, 91-102 (2016)

B. Mason, The enstatite chondrites. Geochim. Cosmochim. Acta 30, 23-39 (1966)

K.J. Mathew, K. Marti, Early evolution of Martial volatiles: nitrogen and noble gas components in ALH84001 and Chassigny. J. Geophys. Res. 106, 1401-1422 (2001)

B. Mayer, N. Wittig, M. Humayun, I. Leya, Palladium isotopic evidence for nucleosynthetic and cosmogenic isotope anomalies in IVB iron meteorites. Astrophys. J. 809, 180-188 (2015)

W.F. McDonough, S.-S. Sun, The composition of the Earth. Chem. Geol. 120, 223-253 (1995)

R. Meijerink, K.M. Pontoppidan, G.A. Blake, D.R. Poelman, C.P. Dullemond, Radiative transfer models ofmid-infrared H2O lines in the planet-forming region of circumstellar disks. Astrophys. J. 704, 1471- 1481 (2009)

S. Mikhail, E. Füri, On the origin(s) and evolution of Earth's carbon. Elements 15, 307-312 (2019)

A. Morbidelli, B. Bitsch, A. Crida, M. Gounelle, T. Guillot, S. Jacobson, A. Johansen, M. Lambrechts, E. Lega, Fossilized condensation lines in the Solar System protoplanetary disk. Icarus 267, 368-376 (2016)

A. Morbidelli, K.J. Walsh, D.P. O'Brien, D.A. Minton, W.F. Bottke, Dynamical evolution of the asteroid belt, in Asteroids $I V$, ed. by P. Michel, F. DeMeo, W.F. Bottke (University of Arizona Press, Tucson, 2015), pp. 493-508

A. Morbidelli, J.I. Lunine, D.P.O. Brien, S.N. Raymond, K.J. Walsh, Building terrestrial planets. Annu. Rev. Earth Planet. Sci. 40, 251278 (2012)

N. Moskovitz, E. Gaidos, Differentiation of planetesimals and the thermal consequences of melt migration. Meteorit. Planet. Sci. 46, 903-918 (2011)

H. Nakano, A. Kouchi, S. Tachibana, A. Tsuchiyama, Evaporation of interstellar organic materials in the solar nebula. Astrophys. J. 592, 1252-1262 (2003) 
H. Nakano, N. Hirakaw, Y. Matsubara, S. Yamashita, T. Okuchi, K. Asahina, R. Tanaka, N. Suzuki, H. Naraoka, Y. Takano, S. Tachibana, T. Hama, Y. Oba, Y. Kimura, N. Watanabe, A. Kouchi, Precometaryorganic matter: a hidden reservoir of water inside the snow line. Sci. Rep. 10, 7755 (2020)

J.A.M. Nanne, F. Nimmo, J.N. Cuzzi, T. Kleine, Origin of the non-carbonaceous-carbonaceous meteorite dichotomy. Earth Planet. Sci. Lett. 511, 44-54 (2019)

S. Niemeyer, Titanium isotope anomalies in chondrules from carbonaceous chondrites. Geochim. Cos- mochim. Acta 52, 309-318 (1988)

L.R. Nittler, F. Ciesla, Astrophysics with extraterrestrial materials. Annu. Rev. Astron. Astrophys. 54, 53-93 (2016)

A. Oka, T. Nakamoto, S. Ida, Evolution of snow line in optically thick protoplanetary disks: effect of water ice opacity and dust grain size. Astrophys. J. 738, 141 (2011)

U. Ott, Planetary and pre-solar noble gases in meteorites. Chem. Erde 74, 519-544 (2014)

H. Palme, H. O’Neill, Cosmochemical estimates of mantle composition, in The Mantle and Core, vol. 3, ed.by R.W. Carlson (2003), pp. 1-39. Treatise on Geochemistry, vol. 3, 2nd edn., ed. by H.D. Holland, K.K. Turekian (Elsevier)

H. Palme, K. Lodders, A. Jones, Solar system abundances of the elements, in Treatise on Geochemistry, vol. 2, ed. by H.D. Holland, K.K. Turekian 2nd edn. (Elsevier, Oxford, 2014), pp. 15-36

M. Palot, P. Cartigny, J.W. Harris, F.V. Kaminsky, T. Stachel, Evidence for deep mantle convection and primordial heterogeneity from nitrogen and carbon stable isotopes in diamond. Earth Planet. Sci. Lett. 357-358, 179-193 (2012)

J. Pape, K. Mezger, A.-S. Bouvier, L.P. Baumgartner, Time and duration of chondrule formation: constraints from ${ }^{26} \mathrm{Al}-{ }^{26} \mathrm{Mg}$ ages of individual chondrules. Geochim. Cosmochim. Acta 244, 416-436 (2019)

A. Patzer, L. Schultz, Noble gases in enstatite chondrites II: the trapped component. Meteorit. Planet. Sci. 37, 601-612 (2002)

V.K. Pearson, M.A. Sephton, I. Gilmour, I. Franchi, Hydrogen isotopic composition of the Tagish Lake me- teorite: comparison with other carbonaceous chondrites, in 32nd Annual Lunar and Planetary Science Conference, Abstract 1861 (2001)

V.K. Pearson, M.A. Sephton, I.A. Franchi, J.M. Gibson, I. Gilmour, Carbon and nitrogen in carbonaceous chondrites: elemental abundances and stable isotopic compositions. Meteorit. Planet. Sci. 41, 1899- 1918 (2006)

L. Piani, F. Robert, L. Remusat, Micron-scale D/H heterogeneity in chondrite matrices: a signature of the pristine solar system water? Earth Planet. Sci. Lett. 415, 154-164 (2015)

L. Piani, Y. Marrocchi, T. Rigaudier, L.G. Vacher, D. Thomassin, B. Marty, Earth's water may have been inherited from material similar to enstatite chondrite meteorites. Science 369, 1110-1113 (2020).

G.M. Poole, M. Rehkämper, B.J. Coles, T. Goldberg, C.L. Smith, Nucleosynthetic molybdenum isotope anomalies in iron meteorites new evidence for thermal processing of solar nebula material. Earth Planet. Sci. Lett. 473, 215-226 (2017)

L. Qin, R.W. Carlson, Nucleosynthetic isotope anomalies and their cosmochemical significance. Geochem. J. 50, 43-65 (2016)

S.N. Raymond, D.P. O'Brien, A. Morbidelli, N.A. Kaib, Building the terrestrial planets: constrained accretion in the inner solar system. Icarus 203, 644-662 (2009)

S.N. Raymond, A. Izidoro, Origin of water in the inner solar system: planetesimals scattered inward during Jupiter and Saturn's rapid gas accretion. Icarus 297, 134-148 (2017)

S.N. Raymond, A. Izidoro, A. Morbidelli, Solar system formation in the context of extrasolar planets, in Plan- etary Astrobiology, ed. by V. Meadows, G.N. Arney, B.E. Schmidt D.J. Marais (University of ArizonaPress, Tucson, 2020), pp. 287-324

J. Render, S. Ebert, C. Burkhardt, T. Kleine, G.A. Brennecka, Titanium isotopic evidence for a shared genetic heritage of refractory inclusions from different carbonaceous chondrites. Geochim. Cosmochim. Acta 254, 40-53 (2019)

J.H. Reynolds, G. Turner, Rare gases in chondrite Renazzo. J. Geophys. Res. 49, 3263-3281 (1964)

F. Robert, The D/H ratio in chondrites. Space Sci. Rev. 106, 87-101 (2003)

F. Robert, S. Epstein, The concentration and isotopic composition of hydrogen, carbon and nitrogen in car- bonaceous meteorites. Geochim. Cosmochim. Acta 46, 81-95 (1982)

D.C. Rubie, S.A. Jacobson, A. Morbidelli, D.P. O’Brien, E.D. Young, J. de Vries, F. Nimmo, H. Palme, D.J. Frost, Accretion and differentiation of the terrestrial planets with implications for the composition of early-formed Solar System bodies. Icarus 248, 89$108(2015)$

A.E. Rubin, B. Fegley, R. Brett, Oxidation state in chondrites, in Meteorites and the Early Solar System, ed. by J.F. Kerridge, M.S. Mathews (University of Arizona Press, Tucson, 1988), pp. 488-511

A.E. Rubin, B.-G. Choi, Origin of halogens and nitrogen in enstatite chondrites. Earth Moon Planets 105, 41-53 (2009)

A.E. Rubin, J.T. Wasson, Variations of chondrite properties with heliocentric distance. Meteoritics 30, 569 (1995) (Abstr.)

A.E. Rubin, C. Ma, Meteoritic minerals and their origins. Geochemistry 77, 325-385 (2017)

S. Sahijpal, P. Soni, G. Gupta, Numerical simulations of the differentiation of accreting planetesimals with ${ }^{26} \mathrm{Al}$ and ${ }^{60} \mathrm{Fe}$ as the heat sources. Meteorit. Planet. Sci. 42, 1529-1548 (2007)

D.D. Sasselov, M. Lecar, On the snow line in dusty protoplanetary disks. Astrophys. J. 528, 995-998 (2000)

T. Sato, S. Okuzumi, S. Ida, On the water delivery to terrestrial embryos by ice pebble accretion. Astron. Astrophys. 589, A15 (2016)

E.R.D. Scott, Formation of olivine-metal textures in pallasite meteorites. Geochim. Cosmochim. Acta 41, 693-710 (1977)

E.R.D. Scott, A.N. Krot, Chondritic meteorites and the high-temperature nebular origins of their components, in Chondrites and the Protoplanetary Disk, ed. by A.N. Krot et al. ASP Conference Series, vol. 341 (Astronomical Society of the Pacific, San Francisco, 2005), pp. 15-52

E.R.D. Scott, A.N. Krot, I.S. Sanders, Isotopic dichotomy among meteorites and its bearing on the protoplan- etary disk. Astrophys. J. 854, 164 (2018)

F.H. Shu, F.C. Adams, S. Lizano, Star formation in molecular clouds: observation and theory. Annu. Rev. Astron. Astrophys. 25, 23-72 
(1987)

F.H. Shu, X-rays and fluctuating X-winds from protostars. Science 277, 1475-1479 (1997)

F.H. Shu, H. Shang, M. Gounelle, A.E. Glassgold, T. Lee, The origin of chondrules and refractory inclusions in chondritic meteorites. Astrophys. J. 548, 1029-1050 (2001)

A. Shukolyukov, G.W. Lugmair, Live iron-60 in the early solar system. Science 259, 1138-1142 (1993a)

A. Shukolyukov, G.W. Lugmair, ${ }^{60} \mathrm{Fe}$ in eucrites. Earth Planet. Sci. Lett. 119, 159-166 (1993b)

F. Spitzer, C. Burkhardt, G. Budde, T.S. Kruijer, A. Morbidelli, T. Kleine, Isotopic evolution of the inner Solar System inferred from molybdenum isotopes in meteorites. Astrophys. J. Lett. 898, L2 (2020)

T. Stephan, A.M. Davis, Molybdenum in presolar silicon carbide grains reveal details of s-, r-, and p-process nucleosynthesis, in $82 n d$ Annual Meeting of the Meteoritical Society (2019). LPI Contrib. No. 2157, abstract \#6107

A. Stephant, L.A.J. Garvie, P. Mane, R. Hervig, M. Wadhwa, Terrestrial exposure of a fresh Martian meteorite causes rapid changes in hydrogen isotopes and water concentrations. Sci. Rep. 8, 12385 (2018)

N. Sugiura, W. Fujiya, Correlated accretion ages and $\varepsilon^{54} \mathrm{Cr}$ of meteorite parent bodies and the evolution of the solar nebula. Meteorit. Planet. Sci. 49, 772-787 (2014)

S. Sutton, C.M.O'D. Alexander, A. Bryant, A. Lanzirotti, M. Newville, E.A. Cloutis, The bulk valence state of Fe and the origin of water in chondrites. Geochim. Cosmochim. Acta 211, 115-132 (2017)

S.R. Taylor, Solar System Evolution, 2nd edn. (Cambridge University Press, Cambridge, 2001). 484 pp.

A. Takigawa, R.M. Stroud, L.R. Nittler, C.M.O'D. Alexander, A. Miyake, High-temperature dust condensa- tion around an AGB star: evidence from a highly pristine presolar corundum. Astrophys. J. 862, L13 (2018)

M.H. Thiemens, J.E. Heidenreich, The mass independent fractionation of oxygen: a novel isotope effect and its possible cosmochemical implications. Science 219, 1073-1075 (1983)

Z.A. Torrano, G.A. Brennecka, C.D. Williams, S.J. Romaniello, V.K. Rai, R.R. Hines, M. Wadhwa, Titanium isotope signatures of calcium-aluminum-rich inclusions from $\mathrm{CV}$ and $\mathrm{CK}$ chondrites: implications for early Solar System reservoirs and mixing. Geochim. Cosmochim. Acta 263, 13-30 (2019)

J.M. Trigo-Rodríguez, A. Rimola, S. Tanbakouei, V. Cabedo Soto, M. Lee, Accretion of water in carbona- ceous chondrites: current evidence and implications for the delivery of water to early Earth. Space Sci.Rev. 215, 18 (2019)

A. Trinquier, J.L. Birck, C.J. Allègre, Widespread ${ }^{54} \mathrm{Cr}$ heterogeneity in the inner solar system. Astrophys. J. 655, 1179-1185 (2007)

A. Trinquier, T. Elliott, D. Ulfbeck, C. Coath, A.N. Krot, M. Bizzarro, Origin of nucleosynthetic isotope heterogeneity in the Solar protoplanetary disk. Science 324, 374-376 (2009)

H.C. Urey, The Planets: Their Origin and Development (Yale University Press, New Haven, 1952), 245 pp.

L. Vacher, L. Piani, T. Rigaudier, D. Thomassin, G. Florin, M. Piralla, Y. Marrocchi, Hydrogen in chondrites: influence of parent body alteration and atmospheric contamination on primordial components. Geochim. Cosmochim. Acta 281, 53-66 (2020)

P. Vernazza, B. Zanda, R.P. Binzel, T. Hiroi, F.E. DeMeo, M. Birlan, R. Hewins, L. Ricci, P. Barge, M. Lockhart, Multiple and fast: the accretion of ordinary chondrite parent bodies. Astrophys. J. 791, 120-144 (2014)

P. Vernazza, M. Marsset, P. Beck, R.P. Binzel, M. Birlan, E.A. Cloutis, F.E. DeMeo, C. Dumas, T. Hiroi, Compositional homogeneity of CM parent bodies. Astrophys. J. 152, 54-64 (2016)

J. Villeneuve, M. Chaussidon, G. Libourel, Homogeneous distribution of ${ }^{26} \mathrm{Al}$ in the Solar System from the Mg isotopic composition of chondrules. Science 325, 985-988 (2009)

R. Visser, E.F. van Dishoeck, S.D. Doty, C.P. Dullemond, The chemical history of molecules in circumstellar disks. Astron. Astrophys. 495, 881-897 (2009)

R.J. Walker, Highly siderophile elements in the Earth, Moon and Mars: update and implications for planetary accretion and differentiation. Geochemistry 69, 101-125 (2009)

R.J. Walker, K. Bermingham, J. Liu, I.S. Puchtel, M. Touboul, E.A. Worsham, In search of late-stage plane- tary building blocks. Chem. Geol. 411, 125-142 (2015)

N.P. Walte, G.F.D. Solferino, G.J. Golabek, D.S. Souza, A. Bouvier, Two-stage formation of pallasites and the evolution of their parent bodies revealed by deformation experiments. Earth Planet. Sci. Lett. 546, 116419 (2020)

K.J. Walsh, A. Morbidelli, S.N. Raymond, D.P. O’Brien, A.M. Mandell, A low mass for Mars from Jupiter's early gas-driven migration. Nature 475, 206-209 (2011)

K.J. Walsh, H.F. Levison, Planetesimals to terrestrial planets: collisional evolution amidst a dissipating gas disk. Icarus 329, 88-100 (2019)

H. Wang, R.C. Bell, M.J. Iemeda, A.A. Tsekouras, J.P. Cowin, Sticky ice grains aid planet formation: unusual properties of cryogenic water ice. Astrophys. J. 620, 1028-1032 (2005)

P.H. Warren, Stable-isotopic anomalies and the accretionary assemblage of the Earth and Mars: a subordinate role for carbonaceous chondrites. Earth Planet. Sci. Lett. 311, 93-100 (2011)

J.T. Wasson, Meteorites: Their Record of Early Solar-System History (W.H. Freeman, New York, 1985)

J.T. Wasson, The building stones of the planets, in Mercury, ed. by F. Vilas, C.R. Chapman, M.S. Matthews (University of Arizona Press, Tucson, 1988), pp. 622-650

J.T. Wasson, G.W. Kallemeyn, Compositions of chondrites. Philos. Trans. R. Soc. A, Math. Phys. Eng. Sci. 32, 391-403 (1988)

M.K. Weisberg, T.J. McCoy, A.N. Krot, Systematics and evaluation of meteorite classification, in Meteoritesand the Early Solar System II, ed. by D.D. Lauretta, H.Y. McSween (University of Arizona Press, Tucson, 2006), pp. 19-52

C.D. Williams, S. Muhkopadyay, Capture of nebular gases during Earth's accretion is preserved in deep- mantle neon. Nature 565, 78- 
$81(2018)$

J.P. Williams, L.A. Cieza, Protoplanetary disks and their evolution. Annu. Rev. Astron. Astrophys. 49, 67-117 (2011)

J.A. Wood, The chondrite types and their origins, in Chondrites and the Protoplanetary Disk, ed. by A.N. Krot, E.R.D. Scott, B. Reipurth (Astronomical Society of the Pacific, Hawai'i, 2005), pp. 953-971

B.J. Wood, J. Li, A. Shahar, Carbon in the core: its influence on the properties of core and mantle. Rev. Mineral. Geochem. 75, 231-250 (2013)

E.A. Worsham, K.R. Bermingham, R.J. Walker, Characterizing cosmochemical materials with genetic affini- ties to the Earth: genetic and chronological diversity within the IAB iron meteorite complex. Earth Planet. Sci. Lett. 467, 157-166 (2017)

J. Wu, S.J. Desch, L. Schaefer, L.T. Elkins-Tanton, K. Pahlevan, P.R. Buseck, Origin of Earth's water: chon- dritic inheritance plus nebular ingassing and storage of hydrogen in the core. J. Geophys. Res., Planets123, 2691-2712 (2018)

L. Yang, F.J. Ciesla, The effects of disk building on the distributions of refractory materials in the solar nebula. Meteorit. Planet. Sci. 47, 99-119 (2012)

J. Yang, J.I. Goldstein, E.R.D. Scott, Iron meteorite evidence for early formation and catastrophic disruption of protoplanets. Nature 446, 888-891 (2007)

J. Yang, J.I. Goldstein, E.R.D. Scott, Main-group pallasites: thermal history, relationship to IIIAB iron, and origin. Geochim. Cosmochim. Acta 74, 4471-4492 (2010)

R. Yokochi, B. Marty, A determination of the neon isotopic composition of the deep mantle. Earth Planet. Sci. Lett. 225, 77-88 (2004)

T. Yokoyama, Y. Nagai, R. Fukai, T. Hirata, Origin and evolution of distinct molybdenum isotopic variabilities within carbonaceous and non-carbonaceous reservoirs. Astrophys. J. 883, 62 (2019)

J. Zhang, N. Dauphas, A.M. Davis, I. Leya, A. Fedkin, The proto-Earth as a significant source of lunar material. Nat. Geosci. 5, 251255 (2012)

K. Zhang, G.A. Blake, E.A. Bergin, Evidence of fast pebble growth near condensation fronts in the HL Tau protoplanetary disk. Astrophys. J. Lett. 806, L7 (2015)

E. Zinner, Presolar grains, in Meteorites and Cosmochemical Processes, ed. by A.M. Davis (Elsevier, New York, 2014), pp. 181-213. Treatise on Geochemistry, vol. 1, 2nd edn., ed. by H.D. Holland, K.K. Turekian

E. Zinner, M. Tang, E. Anders, Nature 330, 730-732 (1987)

E. Zinner, S. Amari, R. Guinness, C. Jennings, A.F. Mertz, A.N. Nguyen, R. Gallino, P. Hoppe, M. Lugaro, L.R. Nittler, R.S. Lewis, NanoSIMS isotopic analysis of small presolar grains: search for Si3N4 grains from AGB stars and Al and $\mathrm{Ti}$ isotopic compositions of rare presolar SiC grains. Geochim. Cosmochim. Acta 71, 4786-4813 (2007) 